\title{
Coupling of Challenging Heteroaryl Halides with Alkyl Halides via Nickel-Catalyzed Cross-Electrophile Coupling
}

\author{
Eric C. Hansen, ${ }^{* \dagger}, \| \odot$ Changfeng Li, ${ }^{\ddagger}, \|$ Sihang Yang, Dylan Pedro, ${ }^{\dagger}$ and Daniel J. Weix ${ }^{*}, \S \odot$ \\ ${ }^{\dagger}$ Chemical Research and Development Pfizer Worldwide Research and Development, Eastern Point Road, Groton, Connecticut \\ 06340, United States \\ ${ }^{\ddagger}$ Asymchem Life Science (Tianjin) Co., Ltd., 71 Seventh Avenue, TEDA, Tianjin 300457, China \\ ${ }^{\S}$ Department of Chemistry, University of Rochester, Rochester, New York 14627-0216, United States
}

\section{Supporting Information}

ABSTRACT: Despite their importance, the synthesis of alkylated heterocycles from the cross-coupling of Lewis basic nitrogen heteroaryl halides with alkyl halides remains a challenge. We report here a general solution to this challenge enabled by a new collection of ligands based around 2-pyridyl- $N$-cyanocarboxamidine and 2-pyridylcarboxamidine cores. Both primary and secondary alkyl halides can be coupled with 2-, 3-, and 4-pyridyl halides as well as other more complex heterocycles in generally good yields (41 examples, 69\% ave yield).

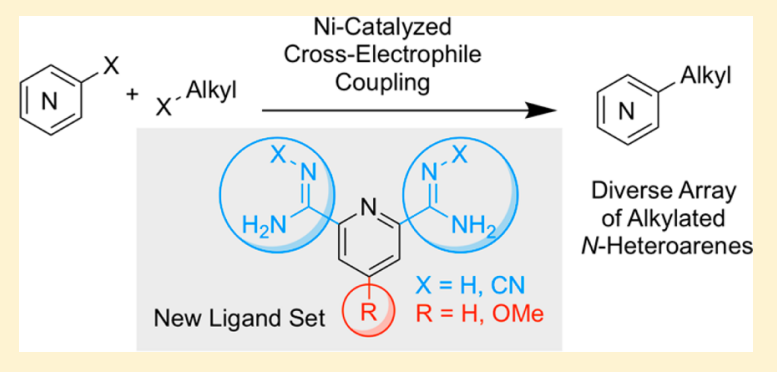

\section{INTRODUCTION}

Nitrogen heterocycles are common structures in pharmaceutical chemistry but often present challenges in transition-metalcatalyzed cross-coupling reactions. ${ }^{1}$ While great advancements have been made in cross-coupling to make aryl-heteroaryl bonds $\left(\mathrm{Csp}^{2}-\mathrm{Csp}^{2}\right)$, the corresponding synthesis of heteroaryl-alkyl bonds $\left(\mathrm{Csp}^{2}-\mathrm{Csp}^{3}\right)$, while desired by industry, remains challenging. ${ }^{1}$ Strategies starting from heteroaryl nucleophiles or alkyl nucleophiles have been examined in detail, ${ }^{2}$ but the carbon nucleophile reagents can limit functional group compatibility, and fewer are commercially available than carbon electrophiles. Substrate-directed strategies, like Minisci chemistry ${ }^{3}$ or $\mathrm{C}-\mathrm{H}$ functionalization, ${ }^{2 \mathrm{~b}, 4}$ can avoid the need for a prefunctionalized heteroarene, but the regiochemistry of the alkylation is dictated by the structure of the heteroarene. A useful compromise solution is the cross-coupling of two different electrophiles. Cross-electrophile coupling ${ }^{5}$ avoids the need for a carbon nucleophile, but reactions of heteroaryl halides with alkyl halides have not been extensively explored. ${ }^{6-8}$ The majority of examples with unactivated alkyl halides have been with simple 2-halopyridines, while Reisman has reported the coupling of mostly 3-halopyridines (and pyrimidines) with activated alkyl halides. ${ }^{7}$ A generalized approach with unactivated alkyl halides has not yet been realized but would greatly benefit drug discovery and development. ${ }^{9}$

Previously reported advances involved modification of the solvent and the use of additives but largely utilized the same small number of bipyridine and phenanthroline ligands that have been employed for simple aryl halides. This small ligand pool has been a limitation not only of reductive crosselectrophile coupling but also related photoredox crosscoupling $^{10}$ and cross-couplings of redox active esters. ${ }^{11}$
We recently reported a new approach to ligand discovery that uncovered pyridyl carboxamidines (1-6, Scheme 1) as

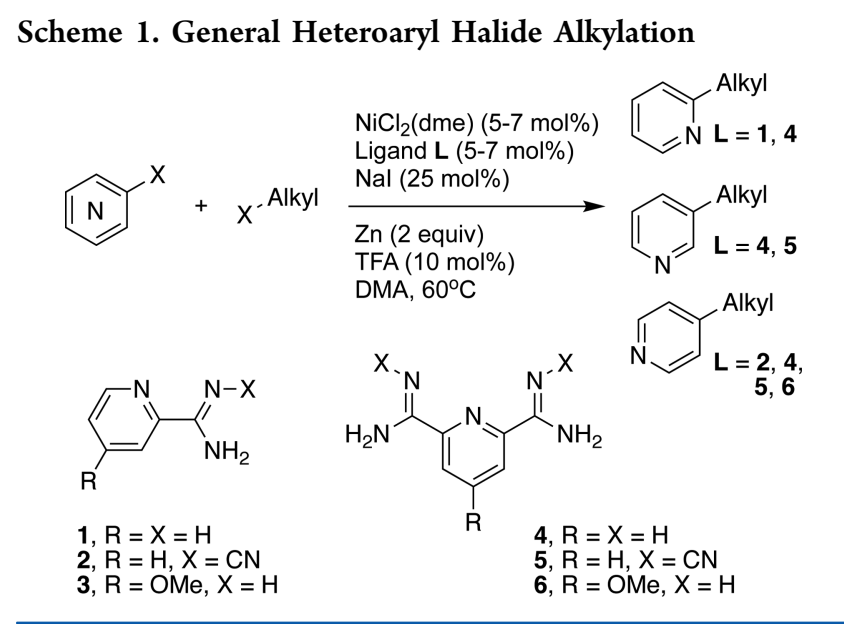

excellent ligands for cross-electrophile coupling. ${ }^{12}$ In that study, the only tridentate ligand examined was $4,^{13}$ and only 3bromopyridines were examined. We considered that a slightly expanded set of ligands might allow a broad array of heteroaryl halides to be coupled. Herein, we show that a small collection of pyridyl carboxamidine ligands suffices for the cross-coupling of a wide variety of basic nitrogen heterocycles with primary and secondary alkyl halides (Scheme 1).

Received: May 30, 2017

Published: July 6, 2017 


\section{RESULTS AND DISCUSSION}

We began by examining substituted 4-halopyridines using a common alkyl halide (Table 1) using conditions developed

\section{Table 1. Coupling of 4-Bromopyridine Derivatives}

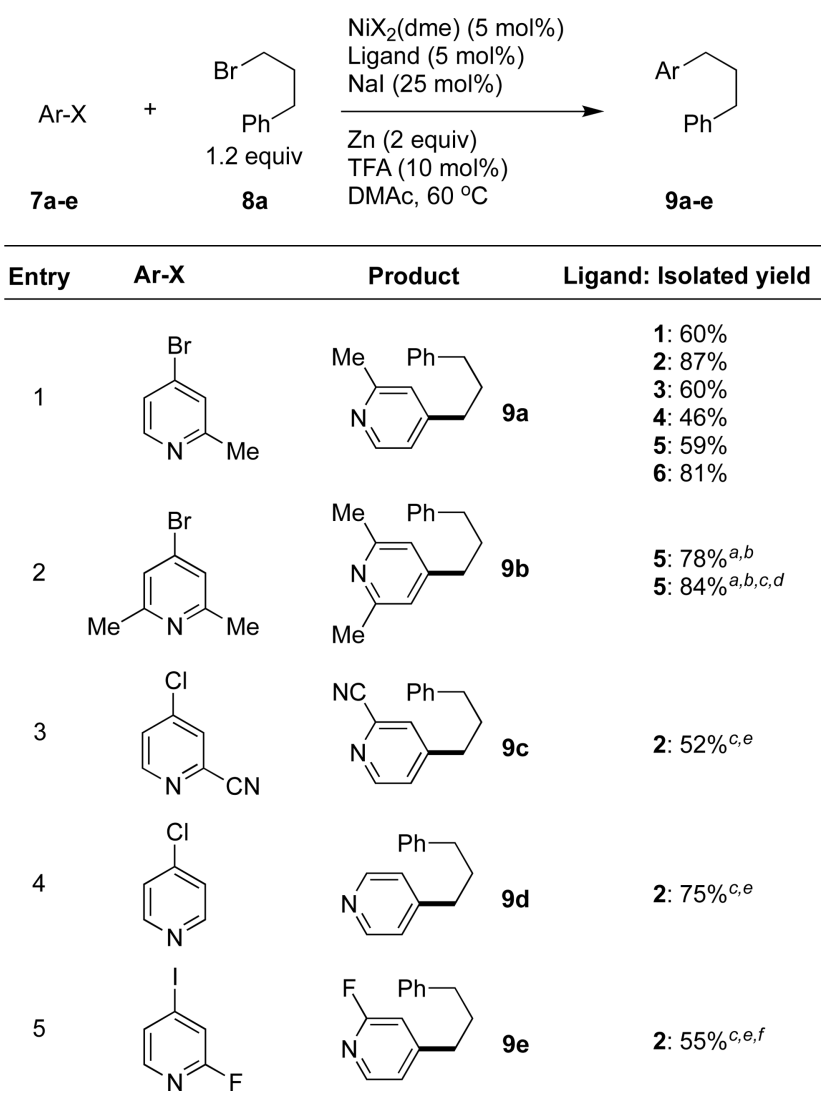

${ }^{a}$ (3-Chloropropyl)benzene (8b) was used instead of $8 \mathbf{a} .{ }^{b}$ Reaction run at $80{ }^{\circ} \mathrm{C} .{ }^{c} 2.0$ equiv of alkyl halide was used. ${ }^{d} 7 \mathrm{~mol} \%$ of $\mathrm{NiCl}_{2}(\mathrm{dme})$ and ligand were used. ${ }^{e} \mathrm{NiI}_{2}(5 \mathrm{~mol} \%)$ was used instead of $\mathrm{NiCl}_{2}$-dme. ${ }^{f} \mathrm{NaI}$ was omitted.

previously by our groups. ${ }^{6 b, 11,14} 1$-Bromo-3-phenylpropane 8 a was chosen due to the presence of a chromophore which allowed analysis of the reaction mixtures by HPLC methods. A survey of the ligands (entry 1) showed that a variety of ligands were effective, including $N$-cyano ligands 2 and 5 and $p$ methoxy-substituted ligand 6. The optimal ligand for this reaction was the bidentate, $\mathrm{N}$-cyano-substituted amidine 2 , prompting us to consider both the bi- and tridentate ligand series in our evaluations. In many reactions, the presence of a large amount of the byproduct arising from alkyl dimerization prompted us to switch from the 1-bromo-3-phenylpropane 8a to 1 -chloro-3-phenylpropane $\mathbf{8 b}$. This tended to minimize the formation of alkyl dimers and produced cross-coupled products in useful yields $(>50 \%)$. This is a rare example of the use of an alkyl chloride in a cross-electrophile coupling reaction and the first example of an unactivated alkyl chloride being coupled with an aryl halide intermolecularly. ${ }^{15}$ These improvements have allowed the successful couplings of 4-chloro-, -bromo-, and -iodopyridines (entries 2-5).

Encouraged by these results, we expanded our survey to include both 3- and 2-halopyridines (Tables 2 and 3, respectively). All of the 3-bromopyridines examined coupled best with ligands 4 and $\mathbf{5}$. While the reaction of simple 3bromopyridine using ligand 4 gave the cross-coupled product in
Table 2. Coupling of 3-Bromopyridine Derivatives

$$
\text { (2) }
$$

$a_{7} \mathrm{~mol} \% \mathrm{NiCl}_{2}(\mathrm{dme})$ and ligand were used. ${ }^{b} 2.0$ equiv of alkyl halide was used. ${ }^{c} \mathrm{NaI}$ was omitted. ${ }_{2}$ equiv of alkyl halide was used at $80{ }^{\circ} \mathrm{C}$.

excellent yield (Table 2, 11a), functionalized 3-bromopyridines generally performed best with the new $\mathrm{N}$-cyano-substituted ligand $5(\mathbf{1 1 b}-\mathbf{f})$. In general, substitution with electrondonating groups gave higher yields, providing cross-coupled products in $80-86 \%$ yield.

Besides 3-halopyridines, several related heterocycles with additional nitrogen atoms also coupled effectively under these conditions $(11 \mathrm{~g}-\mathbf{j})$. This is in contrast to the use of bipyridine ligands, which provided low yields due to competing hydrodehalogenation and dimerization of the heteroaryl halide. While all of these cores are prevalent in the patent literature, we could find only a couple examples of alkylative cross-couplings with 6halo $[1,2,4]$ triazolo $[1,5-a]$ pyridines $(11 \mathrm{~h}, 540$ patents, three references with alkylative cross-coupling), ${ }^{16}$ 3-halo-1,5-naphthyridines (11i, 630 patents, two references with alkylative cross-coupling), ${ }^{17}$ and 6-halo-1-methyl-1H-pyrazolo[4,3-b]pyridines (11j, 264 patents, one reference with alkylative cross-coupling). ${ }^{18}$ The few literature examples all used preformed organometallic reagents (zinc or boron) or enolates 
Table 3. Coupling of 2-Bromopyridine Derivatives

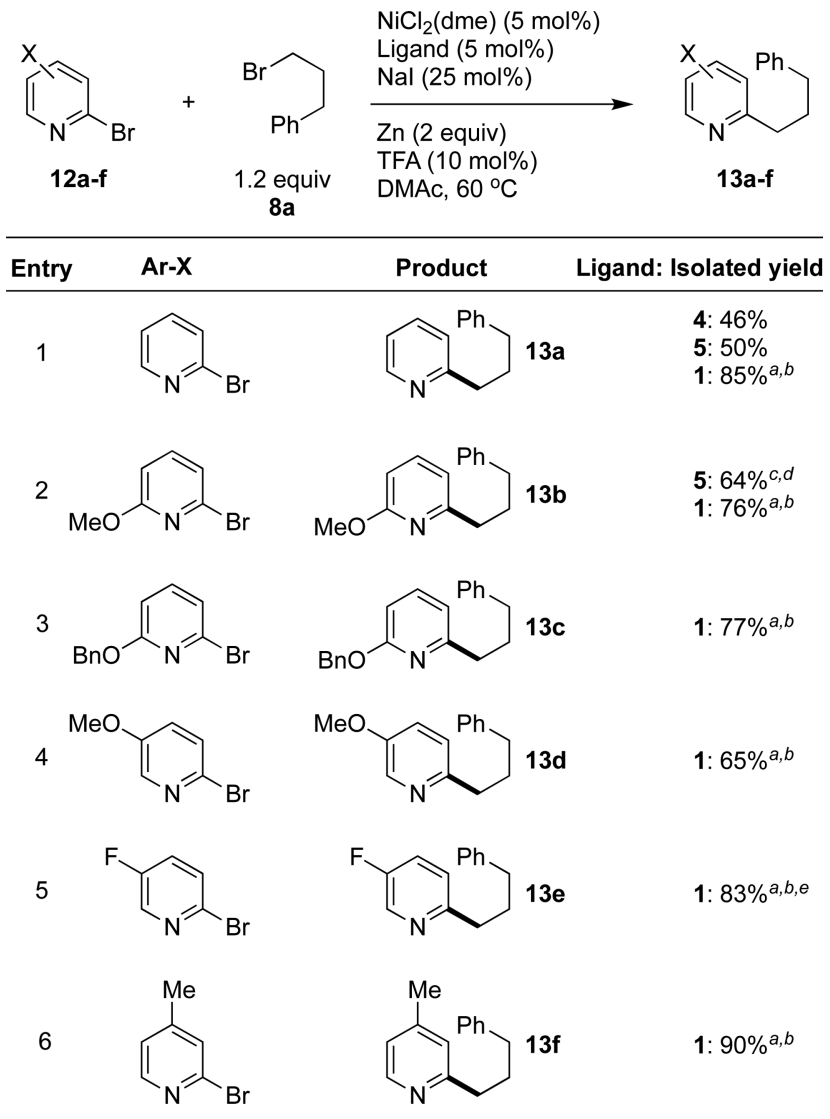

${ }^{a} \mathrm{NiI}_{2}$ was used in place of $\mathrm{NiCl}_{2}(\mathrm{dme}) .{ }^{b} 2$ equiv of 8 a was used. ${ }^{c}(3-$ Chloropropyl)benzene ( $\mathbf{8 b}$ ) was used instead of $\mathbf{8 a}$, and the reaction temperature was $80{ }^{\circ} \mathrm{C} .{ }^{d} 7 \mathrm{~mol} \%$ of $\mathrm{NiCl}_{2}(\mathrm{dme})$ and ligand were used. ${ }^{e}$ Reaction run at $40{ }^{\circ} \mathrm{C}$.

instead of alkyl halides, demonstrating the potential of this new methodology to impact medicinal chemistry.

Successful coupling of pyridines in the 2-position occurred exclusively through the bromopyridine. In contrast to the other pyridines and our previous results, ${ }^{10}$ simple 2-pyridylcarboxamidine 1 was the best ligand. Yields could be further improved by increasing the amount of alkyl bromide to 2 equiv from 1.2 equiv. These results represent a large improvement over the previously reported coupling of 2-chloropyridines. ${ }^{6 \mathrm{~b}}$

To further demonstrate the utility of this reaction, we tested a variety of other substituted primary and secondary alkyl bromide coupling partners with functionality of use in medicinal chemistry (Table 4) using a minimal number of ligands. Synthetically useful yields could be obtained in all cases with ligands 1, 2, and 4, despite the challenging nature of the substrate pairs chosen. Because the optimal ligand for a reaction depends on the structure and reactivity of the alkyl halide as well as the heteroaryl halide, it is difficult to make generalizations. At this time, ligand $\mathbf{4}$ appears to be the most general of the six, and ligand 3, which was superior for difficult aryl bromides in our previous study, ${ }^{11}$ was not among the best in this study of heteroaryl halides.

While isomerization of secondary alkyl groups to form primary alkylated products is a common problem in crosscoupling, ${ }^{19}$ we did not observe any isomerization in the coupling of sec-butyl bromide to form $14 n^{13 a}$

Finally, not all tested heterocycles provided acceptable yields under these conditions: bromopyrazoles and bromoimidazoles
Table 4. Couplings with Functionalized Alkyl Halides
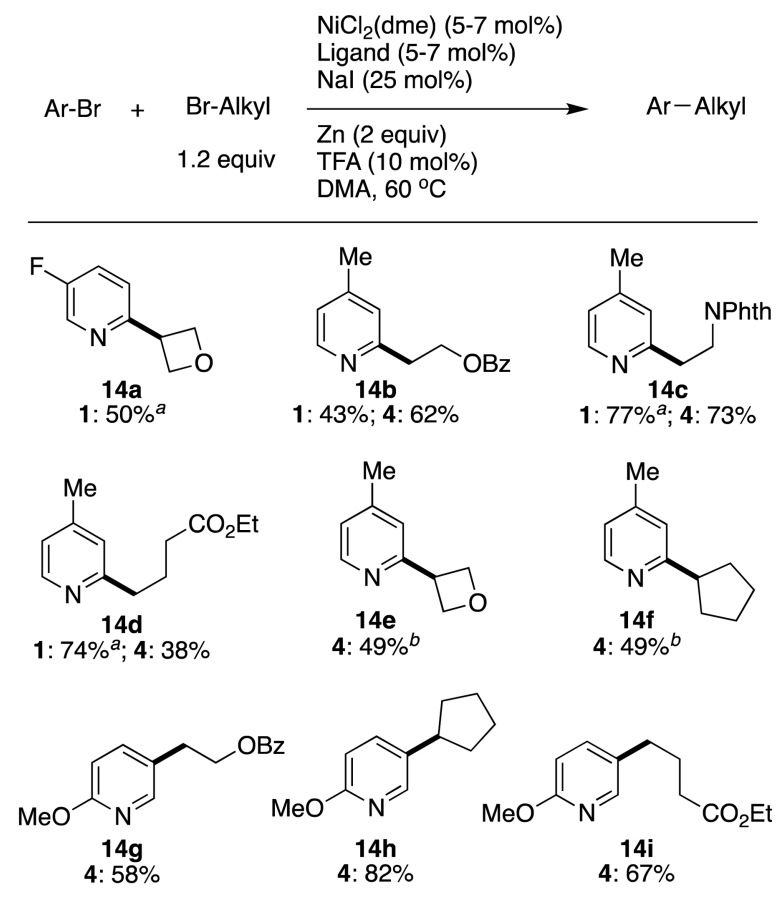<smiles>COc1ccc(CO[Al])cn1</smiles><smiles>CCC(C)c1ccc(OC)nc1</smiles>

${ }^{a} \mathrm{NiI}_{2}$ was used in place of $\mathrm{NiCl}_{2}(\mathrm{dme}) .{ }^{b} 2$ equiv of alkyl halide was used. ${ }^{c}$ Commercial chloromethyl pivalate was used. ${ }^{d} 1.5$ equiv of alkyl halide was used.

provided low yields of the cross-coupled product $(<50 \%)$. At this time, we hypothesize that this difference is related to the differences in the electronics of the corresponding heteroarylnickel(II) complexes $^{13}$ or competing insertion of zinc into the heteroaryl-X bond. ${ }^{20}$

\section{CONCLUSIONS}

This work, along with our initial report, ${ }^{10}$ shows that three to six ligands suffice to couple a broad range of aryl and heteroaryl halides with alkyl halides under a standard set of conditions. Notably, this work demonstrates that $\mathrm{N}$-cyanocarboxamidine ligands can, in some cases, be superior to unsubstituted carboxamidine ligands. We anticipate that these catalysts will also prove useful for the growing number of reactions that use 
bipyridine-ligated nickel catalysts. ${ }^{9}$ Studies to understand why these $N$-cyanocarboxamidine catalysts perform better are ongoing. $^{21}$

\section{EXPERIMENTAL SECTION}

General Information. All starting materials and solvents were purchased from Alfa Aesar, Aldrich, Acros, or TCI chemical companies or from the storehouse of Asychem Laboratories, Inc., and used as received. Ligands 1-4 were synthesized according to the known literature procedures. ${ }^{12}{ }^{1} \mathrm{H}$ and ${ }^{13} \mathrm{C}$ NMR spectra were recorded on Varian Inova 500, Varian Mercury Plus 400, and Varian Mercury Plus 300 instruments, and the chemical shifts $(\delta)$ are expressed in parts per million relative to tetramethylsilane or residual solvent as internal standards. Proton magnetic resonance ( ${ }^{1} \mathrm{H}$ NMR) spectra were recorded at 500 or $400 \mathrm{MHz}$. Carbon magnetic resonance $\left({ }^{13} \mathrm{C} \mathrm{NMR}\right)$ spectra were recorded at 126 or $101 \mathrm{MHz}$. Fluorine magnetic resonance $\left({ }^{19} \mathrm{~F}\right.$ NMR $)$ spectra were recorded at 376 or $282 \mathrm{MHz}$, and the chemical shifts $(\delta)$ are expressed in parts per million relative to $\mathrm{FCCl}_{3}$ as an internal standard. Melting points (mp) were determined using an open melting point capillary and are uncorrected. LC-MS was performed on an Agilent $1260 \mathrm{LC}$ with an Agilent 6230 mass spectrometer (electrospray ionization, ESI) eluting with $0.05 \%$ trifluoroacetic acid in $\mathrm{H}_{2} \mathrm{O}$ and $0.05 \%$ trifluoroacetic acid in $\mathrm{CH}_{3} \mathrm{CN}$.

Synthesis of $(2 Z, 6 Z)-N^{\prime} 2, N^{\prime} 6-D i c y a n o p y r i d i n e-2,6-b i s-$ (carboximidamide) (5). To a $500 \mathrm{~mL}$ flask equipped with magnetic stirbar and $\mathrm{N}_{2}$ purge were added, in order, dry methanol $(200 \mathrm{~mL})$, pyridine-2,6-dicarbonitrile ( $10 \mathrm{~g}, 77 \mathrm{mmol}, 1.0$ equiv), and $20 \mathrm{wt} \%$ sodium methoxide solution in methanol $(2.1 \mathrm{~g}, 7.7 \mathrm{mmol}, 0.10$ equiv). The mixture was stirred for $4 \mathrm{~h}$ before solid cyanamide (95\% potency, $10.3 \mathrm{~g}, 232 \mathrm{mmol}, 3.00$ equiv) was added, and the mixture was allowed to reflux for $6 \mathrm{~h}$. The reaction mixture was then cooled to rt, the slurry was filtered, and the filtrate was dried under a stream of $\mathrm{N}_{2}$ to give crude product. This material was stirred in $200 \mathrm{~mL}$ of hot methanol. The solid was collected on a Büchner funnel and dried on the funnel with an $\mathrm{N}_{2}$ stream to give pure $(2 Z, 6 Z)-N^{\prime} 2, N^{\prime} 6$-dicyanopyridine-2,6bis(carboximidamide) ( $14.5 \mathrm{~g}, 67.8 \mathrm{mmol}, 88 \%$ yield) as a white solid. $\mathrm{Mp}>300{ }^{\circ} \mathrm{C} .{ }^{1} \mathrm{H}$ NMR (401 MHz, DMSO): $\delta 9.94$ (br, $\left.2 \mathrm{H}\right), 9.14$ (br, $2 \mathrm{H}), 8.38(\mathrm{~d}, J=7.9 \mathrm{~Hz}, 2 \mathrm{H}), 8.19(\mathrm{t}, J=7.9 \mathrm{~Hz}, 1 \mathrm{H}) .{ }^{13} \mathrm{C}$ NMR (101 MHz, DMSO): $\delta 164.5,147.4,139.8,125.6,116.1$. HRMS (ESITOF) $(m / z):[\mathrm{M}+\mathrm{H}]^{+}$calcd for $\mathrm{C}_{9} \mathrm{H}_{8} \mathrm{~N}_{7} 214.0841$, found 214.0836 .

Synthesis of 4-Methoxypyridine-2,6-bis(carboximidamide) Dihydrochloride (6). To a $250 \mathrm{~mL}$ flask equipped with magnetic stirbar and $\mathrm{N}_{2}$ purge were sequentially added dry methanol $(200 \mathrm{~mL})$, 4-methoxypyridine-2,6-dicarbonitrile ( $5.0 \mathrm{~g}, 31 \mathrm{mmol}, 1.0$ equiv), and $20 \mathrm{wt} \%$ sodium methoxide solution in methanol $(0.85 \mathrm{~g}, 3.1 \mathrm{mmol}$, 0.10 equiv). The resulting mixture was stirred for $4 \mathrm{~h}$ before ammonium chloride ( $3.3 \mathrm{~g}, 63 \mathrm{mmol}, 2.0$ equiv) was added, and the mixture was stirred at $\mathrm{rt}$ overnight. The resulting slurry was filtered. The filtrate was concentrated to $\sim 5$ volumes and cooled to precipitate the product. This materials was collected on a Büchner funnel and dried under a stream of $\mathrm{N}_{2}$ to give crude product, pyridine-2,6dicarboxamidine, as a white solid. This material was stirred in refluxing ethanol (40 volumes), collected on a Büchner funnel, and again dried under an $\mathrm{N}_{2}$ stream to afford pure 4-methoxypyridine-2,6-dicarboxamidine ( $4.3 \mathrm{~g}, 16 \mathrm{mmol}, 52 \%$ yield). Mp: $248-250{ }^{\circ} \mathrm{C}$ dec: ${ }^{1} \mathrm{H}$ NMR (401 MHz, DMSO): $\delta 10.19$ (br, 4H), $9.79(\mathrm{br}, 4 \mathrm{H}), 8.53(\mathrm{~s}, 2 \mathrm{H})$, $4.09(\mathrm{~s}, 3 \mathrm{H}) .{ }^{13} \mathrm{C}$ NMR (101 MHz, DMSO): $\delta 168.5,160.4,145.3$, 113.7, 57.7. HRMS (ESI-TOF) $(\mathrm{m} / z)$ : of free base $[\mathrm{M}+\mathrm{H}]^{+}$calcd for $\mathrm{C}_{8} \mathrm{H}_{12} \mathrm{~N}_{5} \mathrm{O}$ 194.1042, found 194.1023.

General Procedure for the Nickel-Catalyzed Cross-Electrophile Coupling of Alkyl Halides with Heteroaryl Halides. To a $25 \mathrm{~mL}$ test tube in an EZMax Reactor equipped with a small footballshaped magnetic stirbar and $\mathrm{N}_{2}$ bubbler were added, in the following order, DMAc $(10 \mathrm{~mL}, 20 \mathrm{~mL} / \mathrm{g})$, ligand (0.05 equiv), $\mathrm{NiCl}_{2}(\mathrm{DME})$ ( 0.05 equiv), sodium iodide ( 0.25 equiv), aryl halide $(0.50 \mathrm{~g})$, alkyl halide (1.20 equiv), zinc metal powder $(<10 \mu \mathrm{m}, 2.00$ equiv), and trifluoroacetic acid ( 0.10 equiv). The vial was capped, the atmosphere made inert, and the reaction mixture was heated to $60^{\circ} \mathrm{C}$ with stirring (500 rpm) for several hours. Upon reaction completion (judged by
HPLC or TLC), the reaction mixture was filtered through a Celite pad, and the pad was washed with ethyl acetate $(2 \times 12 \mathrm{~mL})$. The filtrate was then washed with $5 \%$ aq $\mathrm{NH}_{4} \mathrm{OH}(2 \times 20 \mathrm{~mL})$. The organic layer was washed with brine $(20 \mathrm{~mL})$, dried over $\mathrm{MgSO}_{4}$, and filtered to remove the drying agent. The resulting filtrate was then concentrated in vacuo $\left(80 \mathrm{mmHg}, 45{ }^{\circ} \mathrm{C}\right)$ to a residue and diluted with dichloromethane $(3 \mathrm{~mL})$. This solution was wet-loaded onto a silica column for flash chromatographic separation with a gradient between 5 and 30\% ethyl acetate in heptane. The fractions containing product were then concentrated to dryness and analyzed.

2-Methyl-4-(3-phenylpropyl)pyridine (9a). Ligand 1: 17 h, 60\% yield. Ligand 2: 16 h, 87\% yield. Ligand 3: 17 h, $60 \%$ yield. Ligand 4: 29 h, $46 \%$ yield. Ligand 5: 28 h, 59\% yield. Ligand 6: 17 h, $81 \%$ yield. Yellow oil. ${ }^{1} \mathrm{H}$ NMR $\left(500 \mathrm{MHz}, \mathrm{CDCl}_{3}\right): \delta 8.37(\mathrm{~d}, J=5.0 \mathrm{~Hz}, 1 \mathrm{H})$, $7.29(\mathrm{~m}, 2 \mathrm{H}), 7.19(\mathrm{~m}, 3 \mathrm{H}), 6.97(\mathrm{~s}, 1 \mathrm{H}), 6.91(\mathrm{~d}, J=4.7 \mathrm{~Hz}, 1 \mathrm{H})$, $2.65(\mathrm{t}, J=7.6 \mathrm{~Hz}, 2 \mathrm{H}), 2.59(\mathrm{t}, J=7.6 \mathrm{~Hz}, 2 \mathrm{H}), 2.52(\mathrm{~s}, 3 \mathrm{H}), 1.98-$ $1.92(\mathrm{~m}, 2 \mathrm{H}) .{ }^{13} \mathrm{C}$ NMR $\left(126 \mathrm{MHz}, \mathrm{CDCl}_{3}\right): \delta 158.1,151.4,149.0$, $148.8,141.6,128.4,128.3,125.9,125.8,123.4,123.3,121.0,120.9$, 35.2, 34.5, 31.7. HRMS (ESI-TOF) $(m / z):[\mathrm{M}+\mathrm{H}]^{+}$calcd for $\mathrm{C}_{15} \mathrm{H}_{18} \mathrm{~N}$ 212.1439, found 212.1436.

2,6-Dimethyl-4-(3-phenylpropyl)pyridine (9b). Ligand 4: $16 \mathrm{~h}$, $78 \%$ yield (from 2.0 equiv of (3-chloropropyl)benzene); $16 \mathrm{~h}, 84 \%$ yield (from 2.0 equiv of (3-chloropropyl)benzene at $80{ }^{\circ} \mathrm{C}, 7 \mathrm{~mol} \%$ catalyst). Yellow oil. ${ }^{1} \mathrm{H}$ NMR (500 MHz, $\left.\mathrm{CDCl}_{3}\right): \delta 7.29(\mathrm{~m}, 2 \mathrm{H})$, $7.19(\mathrm{~m}, 3 \mathrm{H}), 6.78(\mathrm{~s}, 2 \mathrm{H}), 2.64(\mathrm{t}, J=7.7 \mathrm{~Hz}, 2 \mathrm{H}), 2.55(\mathrm{t}, J=7.7$ $\mathrm{Hz}, 2 \mathrm{H}), 2.48(\mathrm{~s}, 6 \mathrm{H}), 1.96-1.90(\mathrm{~m}, 2 \mathrm{H}) .{ }^{13} \mathrm{C}$ NMR $(126 \mathrm{MHz}$, $\left.\mathrm{CDCl}_{3}\right): \delta 157.5,151.64,141.8,128.4,128.3,125.9,120.4,35.4,34.5$, 31.8, 24.3. HRMS (ESI-TOF) $(m / z):[\mathrm{M}+\mathrm{H}]^{+}$calcd for $\mathrm{C}_{16} \mathrm{H}_{20} \mathrm{~N}$ 226.1596, found 226.1595 .

4-(3-Phenylpropyl)picolinonitrile (9c). Ligand 2: $30 \mathrm{~h}, 52 \%$ yield (from 2.0 equiv of (3-bromopropyl)benzene, $5 \mathrm{~mol} \% \mathrm{NiI}_{2}$ ). Colorless oil. ${ }^{1} \mathrm{H}$ NMR $\left(500 \mathrm{MHz}, \mathrm{CDCl}_{3}\right): \delta 8.58(\mathrm{~d}, J=5.1 \mathrm{~Hz}, 1 \mathrm{H}), 7.51(\mathrm{~s}$, $1 \mathrm{H}), 7.30(\mathrm{~m}, 3 \mathrm{H}), 7.17(\mathrm{~m}, 3 \mathrm{H}), 2.68(\mathrm{~m}, 4 \mathrm{H}), 2.02-1.96(\mathrm{~m}, 2 \mathrm{H})$. ${ }^{13} \mathrm{C}$ NMR $\left(126 \mathrm{MHz}, \mathrm{CDCl}_{3}\right): \delta 152.9,150.9,140.9,133.9,128.6$, $128.5,128.3,127.0,126.2,117.3,35.1,34.2,31.4$. HRMS (ESI-TOF) $(\mathrm{m} / z):[\mathrm{M}+\mathrm{H}]^{+}$calcd for $\mathrm{C}_{15} \mathrm{H}_{15} \mathrm{~N}_{2} 223.1235$, found 223.1223 .

4-(3-Phenylpropyl)pyridine (9d). Ligand 2: 24 h. $75 \%$ yield (from 2.0 equiv of (3-bromopropyl)benzene, $\left.5 \mathrm{~mol} \% \mathrm{NiI}_{2}\right)$. Colorless oil. ${ }^{1} \mathrm{H}$ NMR $\left(500 \mathrm{MHz}, \mathrm{CDCl}_{3}\right): \delta 8.48(\mathrm{~d}, J=4.9 \mathrm{~Hz}, 2 \mathrm{H}), 7.29(\mathrm{~m}, 2 \mathrm{H})$, $7.19(\mathrm{~m}, 3 \mathrm{H}), 7.09(\mathrm{~d}, J=4.9 \mathrm{~Hz}, 2 \mathrm{H}), 2.64(\mathrm{~m}, 4 \mathrm{H}), 1.99-1.93(\mathrm{~m}$, $1 \mathrm{H}) .{ }^{13} \mathrm{C}$ NMR $\left(126 \mathrm{MHz} \mathrm{CDCl}_{3}\right): \delta 151.1149 .6,141.5,128.3$, 125.9, 123.8, 35.2, 34.5, 31.7. HRMS (ESI-TOF) $(m / z):[\mathrm{M}+\mathrm{H}]^{+}$

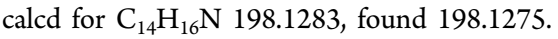

2-Fluoro-4-(3-phenylpropyl)pyridine (9e). Ligand 2: 6 h. 55\% yield (from 2.0 equiv of (3-bromopropyl)benzene, $5 \mathrm{~mol} \% \mathrm{NiI}_{2}$; $\mathrm{NaI}$ is omitted). Yellow oil. ${ }^{1} \mathrm{H}$ NMR (500 MHz, $\left.\mathrm{CDCl}_{3}\right): \delta 8.09$ (d, $J=5.1$ $\mathrm{Hz}, 1 \mathrm{H}), 7.29$ (m, 2H), $7.21(\mathrm{~m}, 1 \mathrm{H}), 7.17(\mathrm{~d}, J=7.5 \mathrm{~Hz}, 2 \mathrm{H}), 6.98$ $(\mathrm{d}, J=4.3 \mathrm{~Hz}, 1 \mathrm{H}), 6.73(\mathrm{~s}, 1 \mathrm{H}), 2.66(\mathrm{t}, J=7.0 \mathrm{~Hz}, 4 \mathrm{H}), 2.00-1.94$ $(\mathrm{m}, 2 \mathrm{H}) .{ }^{13} \mathrm{C}$ NMR $\left(126 \mathrm{MHz}, \mathrm{CDCl}_{3}\right): \delta 164.1(\mathrm{~d}, J=235.6 \mathrm{~Hz})$, $157.3(\mathrm{~d}, J=7.6 \mathrm{~Hz}), 147.3(\mathrm{~d}, J=12.6 \mathrm{~Hz}), 141.3,128.4,128.3$, 126.0, 121.6, 109.1 (d, $J=36.5 \mathrm{~Hz}), 35.2,34.4,31.5 .{ }^{19} \mathrm{~F}$ NMR $(377$ $\left.\mathrm{MHz}, \mathrm{CDCl}_{3}\right): \delta 62.9$. HRMS (ESI-TOF) $(\mathrm{m} / z):[\mathrm{M}+\mathrm{H}]^{+}$calcd for $\mathrm{C}_{14} \mathrm{H}_{15} \mathrm{FN} 216.1189$, found 216.1180 .

3-(3-Phenylpropyl)pyridine (11a). Ligand 4: 19 h, 93\% yield. Ligand 5: $21 \mathrm{~h}, 65 \%$ yield. Pale yellow oil. ${ }^{1} \mathrm{H}$ NMR $(500 \mathrm{MHz}$, $\left.\mathrm{CDCl}_{3}\right): \delta 8.44(\mathrm{~m}, 2 \mathrm{H}), 7.49(\mathrm{~d}, J=7.8 \mathrm{~Hz}, 1 \mathrm{H}), 7.29(\mathrm{t}, J=7.5 \mathrm{~Hz}$, $2 \mathrm{H}), 7.20-7.17(\mathrm{~m}, 4 \mathrm{H}), 2.65(\mathrm{~m}, 4 \mathrm{H}), 1.97(\mathrm{~m}, 2 \mathrm{H}) .{ }^{13} \mathrm{C}$ NMR $(101$ $\left.\mathrm{MHz}, \mathrm{CDCl}_{3}\right): \delta 149.9,147.3,141.6,137.3,135.6,128.3(2), 125.8$, 123.2, 35.2, 32.5, 32.3. HRMS (ESI-TOF) $(\mathrm{m} / z):[\mathrm{M}+\mathrm{H}]^{+}$calcd for $\mathrm{C}_{14} \mathrm{H}_{16} \mathrm{~N}$ 198.1283, found 198.1270.

2-Methoxy-3-(3-phenylpropyl)pyridine (11b). Ligand 5: 70 h, 80\% yield. Colorless oil. ${ }^{1} \mathrm{H}$ NMR $\left(500 \mathrm{MHz}, \mathrm{CDCl}_{3}\right): \delta 8.00(\mathrm{~d}, J=4.2$ $\mathrm{Hz}, 1 \mathrm{H}), 7.33(\mathrm{~d}, J=6.6 \mathrm{~Hz}, 1 \mathrm{H}), 7.26(\mathrm{~m}, 2 \mathrm{H}), 7.17(\mathrm{~m}, 3 \mathrm{H}), 6.77$ $(\mathrm{dd}, J=6.6,4.2 \mathrm{~Hz}, 1 \mathrm{H}), 3.93(\mathrm{~s}, 3 \mathrm{H}), 2.64(\mathrm{t}, J=7.5 \mathrm{~Hz}, 2 \mathrm{H}), 2.59(\mathrm{t}$, $J=7.5 \mathrm{~Hz}, 2 \mathrm{H}), 1.95-1.88(\mathrm{~m}, 2 \mathrm{H}) .{ }^{13} \mathrm{C} \mathrm{NMR}\left(101 \mathrm{MHz}, \mathrm{CDCl}_{3}\right): \delta$ 162.6, 144.6, 142.7, 138.0, 128.8 (2), 126.2, 125.2, 117.0, 53.6, 36.0, 30.9, 30.0. HRMS (ESI-TOF) $(\mathrm{m} / z):[\mathrm{M}+\mathrm{H}]^{+}$calcd for $\mathrm{C}_{15} \mathrm{H}_{18} \mathrm{NO}$ 228.1388 , found 228.1386 .

2-Methoxy-5-(3-phenylpropyl)pyridine (11c). Ligand 5: 18 h, 86\% yield (from 5-iodo-2-methoxypyridine and 2.0 equiv of (3- 
chloropropyl)benzene, $7 \mathrm{~mol} \%$ catalysts; $\mathrm{NaI}$ is omitted); From 5bromo-2-methoxypyridine, $22 \mathrm{~h}, 64 \%$ yield (from 2.0 equiv of (3bromopropyl)benzene at $80{ }^{\circ} \mathrm{C}, 7 \mathrm{~mol} \%$ catalysts). Colorless oil. ${ }^{1} \mathrm{H}$ NMR $\left(401 \mathrm{MHz}, \mathrm{CDCl}_{3}\right): \delta 7.97(\mathrm{~d}, J=2.3 \mathrm{~Hz}, 1 \mathrm{H}), 7.39(\mathrm{dd}, J=$ 8.5, $2.3 \mathrm{~Hz}, 1 \mathrm{H}), 7.28(\mathrm{~m}, 2 \mathrm{H}), 7.18(\mathrm{~m}, 3 \mathrm{H}), 6.68(\mathrm{~d}, J=8.5 \mathrm{~Hz}, 1 \mathrm{H})$, $3.91(\mathrm{~s}, 3 \mathrm{H}), 2.64(\mathrm{t}, J=8.0 \mathrm{~Hz}, 2 \mathrm{H}), 2.56(\mathrm{t}, J=8.0 \mathrm{~Hz}, 2 \mathrm{H}), 1.95-$ $1.87(\mathrm{~m}, 2 \mathrm{H}) .{ }^{13} \mathrm{C}$ NMR $\left(126 \mathrm{MHz}, \mathrm{CDCl}_{3}\right): \delta 162.6,146.0,141.9$, 138.9, 123.0, 128.4, 128.3, 125.8, 110.4, 53.3, 35.1, 32.8, 31.5. HRMS (ESI-TOF) $(\mathrm{m} / z):[\mathrm{M}+\mathrm{H}]^{+}$calcd for $\mathrm{C}_{15} \mathrm{H}_{18} \mathrm{NO} 228.1388$, found 228.1384 .

2-Fluoro-5-(3-phenylpropyl)pyridine (11d). Ligand 5: $18 \mathrm{~h}, 50 \%$ yield (from 2.0 equiv of (3-chloropropyl)benzene at $80{ }^{\circ} \mathrm{C}, 7 \mathrm{~mol} \%$ catalysts). Pale yellow oil. ${ }^{1} \mathrm{H} \mathrm{NMR}\left(401 \mathrm{MHz}, \mathrm{CDCl}_{3}\right): \delta 8.01$ (d, $J=$ $0.7 \mathrm{~Hz}, 1 \mathrm{H}), 7.58(\mathrm{td}, J=8.4,2.5 \mathrm{~Hz}, 1 \mathrm{H}), 7.29(\mathrm{~m}, 2 \mathrm{H}), 7.19(\mathrm{~m}$, $3 \mathrm{H}), 6.84(\mathrm{dd}, J=8.4,2.5 \mathrm{~Hz}, 1 \mathrm{H}), 2.64(\mathrm{~m}, 4 \mathrm{H}), 1.98-1.91(\mathrm{~m}, 2 \mathrm{H})$. ${ }^{13} \mathrm{C}$ NMR $\left(101 \mathrm{MHz}, \mathrm{CDCl}_{3}\right): \delta 162.1(\mathrm{~d}, J=258 \mathrm{~Hz}), 147.0(\mathrm{~d}, J=$ $17.6 \mathrm{~Hz}), 141.5,141.0(\mathrm{~d}, J=10.8 \mathrm{~Hz}), 134.9(\mathrm{~d}, J=1.2 \mathrm{~Hz}), 128.4$, 128.3, 126.0, 109.0 (d, $J=46.6 \mathrm{~Hz}), 35.1,32.6,31.4 .{ }^{19} \mathrm{~F}$ NMR $(377$ $\left.\mathrm{MHz}, \mathrm{CDCl}_{3}\right): \delta$ 59.9. HRMS (ESI-TOF) $(\mathrm{m} / z):[\mathrm{M}+\mathrm{H}]^{+}$calcd for $\mathrm{C}_{14} \mathrm{H}_{15} \mathrm{FN} 216.1189$, found 216.1178 .

5-(3-Phenylpropyl)pyridin-2-yl Di-tert-butylcarbamate (11e). Ligand 5: $24 \mathrm{~h}, 56 \%$ yield (from 2.0 equiv of (3-chloropropyl)benzene at $80{ }^{\circ} \mathrm{C}, 7 \mathrm{~mol} \%$ catalysts). White solid. Mp: $80-82{ }^{\circ} \mathrm{C} .{ }^{1} \mathrm{H}$ NMR $(500$ $\left.\mathrm{MHz}, \mathrm{CDCl}_{3}\right): \delta 8.31(\mathrm{~s}, 1 \mathrm{H}), 7.54(\mathrm{~d}, J=8.3 \mathrm{~Hz}, 1 \mathrm{H}), 7.29(\mathrm{~m}, 2 \mathrm{H})$, 7.20-7.13 (m, 4 H), 2.65 (m, 4H), 1.99-1.93 (m, 2H), $1.43(\mathrm{~s}, 18 \mathrm{H})$. ${ }^{13} \mathrm{C}$ NMR $\left(126 \mathrm{MHz}, \mathrm{CDCl}_{3}\right): \delta 151.4,150.3,148.6,141.6,137.8$, 136.3, 135.7, 128.4, 125.9, 121.6, 82.9, 35.1, 32.5, 31.9, 27.9. HRMS (ESI-TOF) $(m / z):[\mathrm{M}+\mathrm{H}]^{+}$calcd for $\mathrm{C}_{24} \mathrm{H}_{33} \mathrm{~N}_{2} \mathrm{O}_{4} 413.2440$, found 413.2420.

$\mathrm{N}$-(5-(3-Phenylpropyl)pyridin-2-yl)acetamide (11f). Ligand 5: 24 h, $56 \%$ yield (from 2.0 equiv of (3-chloropropyl)benzene at $80{ }^{\circ} \mathrm{C}, 7$ mol \% catalysts). White solid. Mp: $118-120{ }^{\circ} \mathrm{C} .{ }^{1} \mathrm{H}$ NMR $(500 \mathrm{MHz}$, $\left.\mathrm{CDCl}_{3}\right): \delta 9.10(\mathrm{~s}, 1 \mathrm{H}), 8.15(\mathrm{~d}, J=8.2 \mathrm{~Hz}, 1 \mathrm{H}), 8.08(\mathrm{~s}, 1 \mathrm{H}), 7.54(\mathrm{~d}$, $J=8.4 \mathrm{~Hz}, 1 \mathrm{H}), 7.28(\mathrm{~m}, 2 \mathrm{H}), 7.19(\mathrm{~m}, 3 \mathrm{H}), 2.65(\mathrm{t}, J=7.7 \mathrm{~Hz}, 2 \mathrm{H})$, $2.61(\mathrm{t}, J=7.8 \mathrm{~Hz}, 2 \mathrm{H}), 2.19(\mathrm{~s}, 3 \mathrm{H}), 1.94-1.91(\mathrm{~m}, 2 \mathrm{H}) .{ }^{13} \mathrm{C}$ NMR $\left(126 \mathrm{MHz}, \mathrm{CDCl}_{3}\right): \delta 168.7,149.8,147.1141 .6,138.4,133.4,128.3$ (2), 125.9, 114.0, 35.1, 32.6, 31.8, 24.5. HRMS (ESI-TOF) $(\mathrm{m} / z)$ : [M $+\mathrm{H}]^{+}$calcd for $\mathrm{C}_{16} \mathrm{H}_{19} \mathrm{~N}_{2} \mathrm{O}$ 255.1497, found 255.1486.

5-(3-Phenylpropyl)pyrimidine (11g). Ligand 4: $19 \mathrm{~h}, 95 \%$ yield. Colorless oil. ${ }^{1} \mathrm{H}$ NMR $\left(500 \mathrm{MHz}, \mathrm{CDCl}_{3}\right): \delta 9.07(\mathrm{~s}, 1 \mathrm{H}), 8.57(\mathrm{~s}$, $2 \mathrm{H}), 7.30(\mathrm{~m}, 2 \mathrm{H}), 7.19(\mathrm{~m}, 3 \mathrm{H}), 2.69(\mathrm{t}, J=7.6 \mathrm{~Hz}, 1 \mathrm{H}), 2.64(\mathrm{t}, J=$ $7.6 \mathrm{~Hz}, 1 \mathrm{H}), 2.02-1.96(\mathrm{~m}, 2 \mathrm{H}) .{ }^{13} \mathrm{C}$ NMR $\left(101 \mathrm{MHz}, \mathrm{CDCl}_{3}\right): \delta$ 156.7, 156.6, 141.0, 134.9, 128.4, 128.3, 126.0, 35.1, 32.0, 29.7. HRMS (ESI-TOF) $(\mathrm{m} / \mathrm{z}):[\mathrm{M}+\mathrm{H}]^{+}$calcd for $\mathrm{C}_{13} \mathrm{H}_{15} \mathrm{~N}_{2} 199.1235$, found 199.1232.

6-(3-Phenylpropyl) $[1,2,4]$ triazolo[1,5-a]pyridine (11h). Ligand 4: $24 \mathrm{~h}, 74 \%$ yield. Pale yellow oil. ${ }^{1} \mathrm{H}$ NMR $\left(500 \mathrm{MHz}, \mathrm{CDCl}_{3}\right): \delta 8.38$ $(\mathrm{s}, 1 \mathrm{H}), 8.30(\mathrm{~s}, 1 \mathrm{H}), 7.68(\mathrm{~d}, J=9.1 \mathrm{~Hz}, 1 \mathrm{H}), 7.37(\mathrm{dd}, J=9.1,1.4$ $\mathrm{Hz}, 1 \mathrm{H}), 7.30(\mathrm{~m}, 2 \mathrm{H}), 7.20(\mathrm{~m}, 3 \mathrm{H}), 2.70(\mathrm{~m}, 4 \mathrm{H}), 2.05-1.99(\mathrm{~m}$, $2 \mathrm{H}) .{ }^{13} \mathrm{C}$ NMR $\left(126 \mathrm{MHz} \mathrm{CDCl}_{3}\right): \delta 153.4,149.1,141.1,131.3$, 128.2 (2), 128.1, 128.1, 126.1, 125.8, 115.9, 34.8, 31.8, 31.5. HRMS (ESI-TOF) $(\mathrm{m} / z)$ : $[\mathrm{M}+\mathrm{H}]^{+}$calcd for $\mathrm{C}_{15} \mathrm{H}_{16} \mathrm{~N}_{3}$ 238.1344, found 238.1340 .

3-(3-Phenylpropyl)-1,5-naphthyridine (11i). Ligand 4: 17 h, 52\% yield (from 2.0 equiv of (3-chloropropyl)benzene, $7 \mathrm{~mol} \%$ catalysts). Colorless oil. ${ }^{1} \mathrm{H}$ NMR $\left(500 \mathrm{MHz}, \mathrm{CDCl}_{3}\right): \delta 8.96(\mathrm{dd}, J=4.1,1.4$ $\mathrm{Hz}, 1 \mathrm{H}), 8.84(\mathrm{~d}, J=1.8 \mathrm{~Hz}, 1 \mathrm{H}), 8.38(\mathrm{~d}, J=8.4 \mathrm{~Hz}, 1 \mathrm{H}), 8.18(\mathrm{~s}$, $1 \mathrm{H}), 7.60(\mathrm{dd}, J=8.5,4.2 \mathrm{~Hz}, 1 \mathrm{H}), 7.30(\mathrm{~m}, 2 \mathrm{H}), 7.21(\mathrm{~m}, 3 \mathrm{H}), 2.90$ $(\mathrm{t}, J=7.7 \mathrm{~Hz}, 2 \mathrm{H}), 2.73(\mathrm{t}, J=7.6 \mathrm{~Hz}, 2 \mathrm{H}), 2.15-2.08(\mathrm{~m}, 2 \mathrm{H}) .{ }^{13} \mathrm{C}$ NMR $\left(126 \mathrm{MHz}, \mathrm{CDCl}_{3}\right): \delta 152.8,151.1,143.8,142.1,141.4,138.8$, 137.1, 135.4, 128.4, 128.4, 126.0, 123.6, 35.1, 32.5, 32.2. HRMS (ESITOF) $(\mathrm{m} / z):[\mathrm{M}+\mathrm{H}]^{+}$calcd for $\mathrm{C}_{17} \mathrm{H}_{17} \mathrm{~N}_{2}$ 249.1392, found 249.1375 .

1-Methyl-6-(3-phenylpropyl)-1H-pyrazolo[4,3-b]pyridine (11j). Ligand $4: 24 \mathrm{~h}, 51 \%$ yield (from 2.0 equiv of (3-chloropropyl)benzene, $7 \mathrm{~mol} \%$ catalysts). Pale yellow oil. ${ }^{1} \mathrm{H}$ NMR $\left(401 \mathrm{MHz}, \mathrm{CDCl}_{3}\right): \delta$ $8.43(\mathrm{~d}, J=1.4 \mathrm{~Hz}, 1 \mathrm{H}), 8.17(\mathrm{~s}, 1 \mathrm{H}), 7.49(\mathrm{~s}, 1 \mathrm{H}), 7.30(\mathrm{~m}, 2 \mathrm{H}), 7.20$ $(\mathrm{m}, 3 \mathrm{H}), 4.05(\mathrm{~s}, 3 \mathrm{H}), 2.82(\mathrm{t}, J=7.6 \mathrm{~Hz}, 2 \mathrm{H}), 2.70(\mathrm{t}, J=7.6 \mathrm{~Hz}$, $2 \mathrm{H}), 2.05(\mathrm{~m}, 2 \mathrm{H}) .{ }^{13} \mathrm{C}$ NMR $\left(101 \mathrm{MHz}, \mathrm{CDCl}_{3}\right): \delta 147.0,141.5$, 140.3 , 135.0, 133.3, 133.0, 128.5, 128.4, 128.3, 125.9, 115.5, 35.7, 35.3,
32.9. HRMS (ESI-TOF) $(m / z)$ : $[\mathrm{M}+\mathrm{H}]^{+}$calcd for $\mathrm{C}_{16} \mathrm{H}_{18} \mathrm{~N}_{3}$ 252.1501 , found 252.1486

2-(3-Phenylpropyl)pyridine (13a). Ligand 4: $18 \mathrm{~h}, 46 \%$ yield. Ligand 5: $22 \mathrm{~h}, 50 \%$ yield. Ligand $1: 5 \mathrm{~h}, 85 \%$ yield (from 2.0 equiv of (3-bromopropyl)benzene, $5 \mathrm{~mol} \% \mathrm{NiI}_{2}$ ). Colorless oil. ${ }^{1} \mathrm{H}$ NMR (500 $\left.\mathrm{MHz}, \mathrm{CDCl}_{3}\right): \delta 8.53(\mathrm{~d}, J=4.4 \mathrm{~Hz}, 1 \mathrm{H}), 7.57(\mathrm{td}, J=7.7,1.4 \mathrm{~Hz}$, $1 \mathrm{H}), 7.27(\mathrm{~m}, 2 \mathrm{H}), 7.18(\mathrm{~m}, 3 \mathrm{H}), 7.13(\mathrm{~d}, J=7.8 \mathrm{~Hz}, 1 \mathrm{H}), 7.09(\mathrm{dd}, J$ $=7.2,5.2 \mathrm{~Hz}, 1 \mathrm{H}), 2.83(\mathrm{t}, J=7.6 \mathrm{~Hz}, 2 \mathrm{H}), 2.68(\mathrm{t}, J=7.6 \mathrm{~Hz}, 2 \mathrm{H})$, $2.10-2.04(\mathrm{~m}, 2 \mathrm{H}) .{ }^{13} \mathrm{C}$ NMR $\left(126 \mathrm{MHz}, \mathrm{CDCl}_{3}\right): \delta 161.9,149.2$, $142.1,136.3,128.4,128.3,125.7,122.7,121.0,37.9,35.5,31.5$. HRMS (ESI-TOF) $(m / z):[\mathrm{M}+\mathrm{H}]^{+}$calcd for $\mathrm{C}_{14} \mathrm{H}_{16} \mathrm{~N}$ 198.1283, found 198.1278.

2-Methoxy-6-(3-phenylpropyl)pyridine (13b). Ligand 5: 22 h, 64\% yield (from 2.0 equiv of (3-chloropropyl)benzene, $7 \mathrm{~mol} \%$ catalyst). Ligand 1: $20 \mathrm{~h}, 76 \%$ yield (from 2.0 equiv of (3-bromopropyl)benzene, $\left.5 \mathrm{~mol} \% \mathrm{NiI}_{2}\right)$. Colorless oil. ${ }^{1} \mathrm{H}$ NMR $\left(500 \mathrm{MHz} \mathrm{CDCl}_{3}\right): \delta$ $7.44(\mathrm{t}, J=7.7 \mathrm{~Hz}, 1 \mathrm{H}), 7.27(\mathrm{~m}, 2 \mathrm{H}), 7.18(\mathrm{~m}, 3 \mathrm{H}), 6.68(\mathrm{~d}, J=7.2$ $\mathrm{Hz}, 1 \mathrm{H}), 6.53(\mathrm{~d}, J=8.3 \mathrm{~Hz}, 1 \mathrm{H}), 3.92(\mathrm{~s}, 3 \mathrm{H}), 2.72(\mathrm{t}, J=7.6 \mathrm{~Hz}$, $2 \mathrm{H}), 2.67(\mathrm{t}, J=7.7 \mathrm{~Hz}, 2 \mathrm{H}), 2.07(\mathrm{~m}, 2 \mathrm{H}) .{ }^{13} \mathrm{C} \mathrm{NMR}(126 \mathrm{MHz}$, $\left.\mathrm{CDCl}_{3}\right): \delta 159.7,142.4,138.6,128.5,128.2,125.7,115.2,107.3,53.1$, 37.2, 35.4, 30.8. HRMS (ESI-TOF) $(m / z):[\mathrm{M}+\mathrm{H}]^{+}$calcd for $\mathrm{C}_{15} \mathrm{H}_{18} \mathrm{NO} 228.1388$, found 228.1376 .

2-(Benzyloxy)-6-(3-phenylpropyl)pyridine (13c). Ligand 1: $26 \mathrm{~h}$, $77 \%$ yield (from 2.0 equiv of (3-bromopropyl)benzene, $5 \mathrm{~mol} \% \mathrm{NiI}_{2}$ ). Colorless oil. ${ }^{1} \mathrm{H}$ NMR $\left(500 \mathrm{MHz}, \mathrm{CDCl}_{3}\right): \delta 7.45(\mathrm{~m}, 3 \mathrm{H}), 7.35(\mathrm{~m}$, $2 \mathrm{H}), 7.28(\mathrm{~m}, 3 \mathrm{H}), 7.18(\mathrm{~d}, J=7.4 \mathrm{~Hz}, 3 \mathrm{H}), 6.69(\mathrm{~d}, J=7.2 \mathrm{~Hz}, 1 \mathrm{H})$, $6.60(\mathrm{~d}, J=8.2 \mathrm{~Hz}, 1 \mathrm{H}), 5.38(\mathrm{~s}, 2 \mathrm{H}), 2.72(\mathrm{t}, J=7.5 \mathrm{~Hz}, 2 \mathrm{H}), 2.65(\mathrm{t}$, $J=7.5 \mathrm{~Hz}, 2 \mathrm{H}), 2.09-2.03(\mathrm{~m}, 2 \mathrm{H}) .{ }^{13} \mathrm{C}$ NMR $\left(126 \mathrm{MHz} \mathrm{CDCl}_{3}\right): \delta$ $163.1,159.6,142.4,138.7,137.8,128.5,128.3,128.3,128.1,127.6$, $125.7,115.4,107.9,67.3,37.2,35.4,30.8$. HRMS (ESI-TOF) $(\mathrm{m} / z)$ : $[\mathrm{M}+\mathrm{H}]^{+}$calcd for $\mathrm{C}_{21} \mathrm{H}_{21} \mathrm{NO} 304.1701$, found 304.1693.

5-Methoxy-2-(3-phenylpropyl)pyridine (13d). Ligand 1: $22 \mathrm{~h}, 65 \%$ yield (from 2.0 equiv of (3-bromopropyl)benzene, $5 \mathrm{~mol} \mathrm{\%} \mathrm{NiI}_{2}$ ). Colorless oil. ${ }^{1} \mathrm{H}$ NMR $\left(500 \mathrm{MHz}, \mathrm{CDCl}_{3}\right): \delta 8.23(\mathrm{~d}, J=2.4 \mathrm{~Hz}$, $1 \mathrm{H}), 7.26(\mathrm{~m}, 2 \mathrm{H}), 7.17(\mathrm{~m}, 3 \mathrm{H}), 7.10(\mathrm{~m}, 1 \mathrm{H}), 7.03(\mathrm{~d}, J=8.5 \mathrm{~Hz}$, $1 \mathrm{H}), 3.80(\mathrm{~s}, 3 \mathrm{H}), 2.77(\mathrm{t}, J=7.7 \mathrm{~Hz}, 2 \mathrm{H}), 2.66(\mathrm{t}, J=7.7 \mathrm{~Hz}, 2 \mathrm{H})$, $2.06-2.00(\mathrm{~m}, 2 \mathrm{H}) .{ }^{13} \mathrm{C}$ NMR $\left(126 \mathrm{MHz}, \mathrm{CDCl}_{3}\right): \delta 153.88,142.10$, $136.32,128.35,128.17,125.61,122.69,121.12,55.47,55.45,36.73$, 35.39, 31.56. HRMS (ESI-TOF) $(\mathrm{m} / z):[\mathrm{M}+\mathrm{H}]^{+}$calcd for $\mathrm{C}_{15} \mathrm{H}_{18} \mathrm{NO} 228.1388$, found 228.1378 .

5-Fluoro-2-(3-phenylpropyl)pyridine (13e). Ligand 1: 20 h, 83\% yield (from 2.0 equiv of (3-bromopropyl)benzene at $40{ }^{\circ} \mathrm{C}, 5 \mathrm{~mol} \%$ $\mathrm{NiI}_{2}$ ). White solid. Mp: $98-100{ }^{\circ} \mathrm{C}{ }^{1} \mathrm{H}$ NMR $\left(500 \mathrm{MHz}, \mathrm{CDCl}_{3}\right): \delta$ $8.37(\mathrm{~d}, J=2.1 \mathrm{~Hz}, 1 \mathrm{H}), 7.26(\mathrm{~m}, 3 \mathrm{H}), 7.18-7.15(\mathrm{~m}, 3 \mathrm{H}), 7.08(\mathrm{dd}, J$ $=8.5,4.3 \mathrm{~Hz}, 1 \mathrm{H}), 2.80(\mathrm{t}, J=7.7 \mathrm{~Hz}, 2 \mathrm{H}), 2.66(\mathrm{t}, J=7.7 \mathrm{~Hz}, 2 \mathrm{H})$, 2.07-2.01 (m, 2H). ${ }^{13} \mathrm{C}$ NMR $\left(126 \mathrm{MHz}, \mathrm{CDCl}_{3}\right): \delta 157.9(\mathrm{~d}, J=$ $253.3 \mathrm{~Hz}), 157.8(\mathrm{~d}, J=8.8 \mathrm{~Hz}), 141.8,137.1(\mathrm{~d}, J=20.4 \mathrm{~Hz}), 128.3$, $128.2,125.7,123.3,122.9(\mathrm{~d}, J=17.6 \mathrm{~Hz}), 36.9,35.3,31.4 .{ }^{19} \mathrm{~F}$ NMR $\left(377 \mathrm{MHz}, \mathrm{CDCl}_{3}\right): \delta 0.5$. HRMS (ESI-TOF) $(\mathrm{m} / z):[\mathrm{M}+\mathrm{H}]^{+}$calcd for $\mathrm{C}_{14} \mathrm{H}_{15} \mathrm{FN}, 216.1189$, found 216.1179 .

4-Methyl-2-(3-phenylpropyl)pyridine (13f). Ligand 1: 15 h, 90\% yield (from 2.0 equiv of (3-bromopropyl)benzene, $5 \mathrm{~mol} \% \mathrm{NiI}_{2}$ ). Pale yellow oil. ${ }^{1} \mathrm{H}$ NMR $\left(401 \mathrm{MHz}, \mathrm{CDCl}_{3}\right): \delta 8.37(\mathrm{~d}, J=5.0 \mathrm{~Hz}, 1 \mathrm{H})$, $7.26(\mathrm{~m}, 2 \mathrm{H}), 7.20-7.17(\mathrm{~m}, 3 \mathrm{H}), 6.94(\mathrm{~s}, 1 \mathrm{H}), 6.91(\mathrm{~d}, J=5.0 \mathrm{~Hz}$, $1 \mathrm{H}), 2.78(\mathrm{t}, J=8.0 \mathrm{~Hz}, 2 \mathrm{H}), 2.68(\mathrm{t}, J=8.0 \mathrm{~Hz}, 2 \mathrm{H}), 2.30(\mathrm{~s}, 3 \mathrm{H})$, 2.09-2.02 (m, 2H). ${ }^{13} \mathrm{C}$ NMR (101 MHz, $\left.\mathrm{CDCl}_{3}\right): \delta 161.7,149.0$, $147.2,142.2,128.4,128.2,125.7,123.6,122.0,37.8,35.6,31.5,20.9$. HRMS (ESI-TOF) $(m / z):[\mathrm{M}+\mathrm{H}]^{+}$calcd for $\mathrm{C}_{15} \mathrm{H}_{18} \mathrm{~N}$ 212.1439, found 212.1428 .

5-Fluoro-2-(oxetan-3-yl)pyridine (14a). Ligand 1: $14 \mathrm{~h}, 50 \%$ yield ( $\mathrm{NiI}_{2}$ was used in place of $\mathrm{NiCl}_{2}(\mathrm{dme})$ ). White solid. Mp: 118-120 ${ }^{\circ} \mathrm{C}^{1} \mathrm{H}$ NMR $\left(401 \mathrm{MHz}, \mathrm{CDCl}_{3}\right): \delta 8.49(\mathrm{~d}, J=2.8 \mathrm{~Hz}, 1 \mathrm{H}), 7.39(\mathrm{td}$, $J=8.4,2.9 \mathrm{~Hz}, 1 \mathrm{H}), 7.27(\mathrm{dd}, J=8.6,4.3 \mathrm{~Hz}, 1 \mathrm{H}), 5.06(\mathrm{dd}, J=8.4$, $5.9 \mathrm{~Hz}, 2 \mathrm{H}), 4.91(\mathrm{t}, J=6.2 \mathrm{~Hz}, 2 \mathrm{H}), 4.42-4.34(\mathrm{~m}, 1 \mathrm{H}) .{ }^{13} \mathrm{C} \mathrm{NMR}$ $\left(101 \mathrm{MHz}, \mathrm{CDCl}_{3}\right): \delta 158.3(\mathrm{~d}, J=255 \mathrm{~Hz}), 156.2(\mathrm{~d}, J=4.0 \mathrm{~Hz})$, $137.7(\mathrm{~d}, J=23.2 \mathrm{~Hz}), 123.2(\mathrm{~d}, J=18.2 \mathrm{~Hz}), 122.3(\mathrm{~d}, J=4.0 \mathrm{~Hz})$ 77.0, 41.0. ${ }^{19} \mathrm{~F}$ NMR (377 MHz, $\mathrm{CDCl}_{3}$ ): $\delta$ 2.5. HRMS (ESI-TOF) $(m / z):[\mathrm{M}+\mathrm{H}]^{+}$calcd for $\mathrm{C}_{8} \mathrm{H}_{9} \mathrm{FNO} 154.0668$, found 154.0658 .

2-(4-Methylpyridin-2-yl)ethyl Benzoate (14b). Ligand 1: 21 h, 43\% yield. Ligand 4: $10 \mathrm{~h}, 62 \%$ yield. Colorless oil. ${ }^{1} \mathrm{H}$ NMR $(500 \mathrm{MHz}$, 
$\left.\mathrm{CDCl}_{3}\right): \delta 8.41(\mathrm{~d}, J=5.0 \mathrm{~Hz}, 1 \mathrm{H}), 7.99(\mathrm{~d}, J=7.3 \mathrm{~Hz}, 2 \mathrm{H}), 7.53(\mathrm{t}, J$ $=7.6 \mathrm{~Hz}, 1 \mathrm{H}), 7.41(\mathrm{t}, J=7.6 \mathrm{~Hz}, 2 \mathrm{H}), 7.06(\mathrm{~s}, 1 \mathrm{H}), 6.97(\mathrm{~d}, J=5.0$ $\mathrm{Hz}, 1 \mathrm{H}), 4.70(\mathrm{t}, J=6.9 \mathrm{~Hz}, 2 \mathrm{H}), 3.21(\mathrm{t}, J=6.8 \mathrm{~Hz}, 2 \mathrm{H}), 2.31(\mathrm{~s}$, $3 \mathrm{H}) .{ }^{13} \mathrm{C}$ NMR $\left(126 \mathrm{MHz}, \mathrm{CDCl}_{3}\right): \delta 166.4,157.7,149.2,147.4$, $132.8,130.2,129.5,128.2,124.3,122.6,64.1,37.3,20.9$. HRMS (ESITOF) $(\mathrm{m} / z):[\mathrm{M}+\mathrm{H}]^{+}$calcd for $\mathrm{C}_{15} \mathrm{H}_{16} \mathrm{NO}_{2}$ 242.1181, found 242.1170 .

2-(2-(4-Methylpyridin-2-yl)ethyl)isoindoline-1,3-dione (14c). Ligand 1: $15 \mathrm{~h}, 77 \%$ yield $\left(\mathrm{NiI}_{2}\right.$ was used in place of $\left.\mathrm{NiCl}_{2}(\mathrm{dme})\right)$. Ligand 4: $15 \mathrm{~h}, 73 \%$ yield. White solid. Mp: $94-96{ }^{\circ} \mathrm{C} .{ }^{1} \mathrm{H}$ NMR (401 $\left.\mathrm{MHz}, \mathrm{CDCl}_{3}\right): \delta 8.36(\mathrm{~d}, J=5.0 \mathrm{~Hz}, 1 \mathrm{H}), 7.83(\mathrm{dd}, J=5.5,3.0 \mathrm{~Hz}$, $2 \mathrm{H}), 7.70(\mathrm{dd}, J=5.5,3.0 \mathrm{~Hz}, 2 \mathrm{H}), 7.01(\mathrm{~s}, 1 \mathrm{H}), 6.95(\mathrm{~d}, J=5.0 \mathrm{~Hz}$, $1 \mathrm{H}), 4.09(\mathrm{t}, J=8.0 \mathrm{~Hz}, 2 \mathrm{H}), 3.13(\mathrm{t}, J=8.0 \mathrm{~Hz}, 2 \mathrm{H}), 2.29(\mathrm{~s}, 3 \mathrm{H})$. ${ }^{13} \mathrm{C}$ NMR $\left(101 \mathrm{MHz}, \mathrm{CDCl}_{3}\right): \delta 167.9,157.8,149.0,147.3,133.6$, 131.9, 123.9, 123.0, 122.5, 37.6, 36.4, 20.7. HRMS (ESI-TOF) $(\mathrm{m} / z)$ : $[\mathrm{M}+\mathrm{H}]^{+}$calcd for $\mathrm{C}_{16} \mathrm{H}_{15} \mathrm{~N}_{2} \mathrm{O}_{2}$ 267.1134, found 267.1123 .

Ethyl 4-(4-Methylpyridin-2-yl)butanoate (14d). Ligand 1: $21 \mathrm{~h}$, $74 \%$ yield $\left(\mathrm{NiI}_{2}\right.$ was used in place of $\left.\mathrm{NiCl}_{2}(\mathrm{dme})\right)$. Ligand 4: $24 \mathrm{~h}$, $38 \%$ yield. Colorless oil. ${ }^{1} \mathrm{H}$ NMR $\left(500 \mathrm{MHz}, \mathrm{CDCl}_{3}\right): \delta 8.38(\mathrm{~d}, J=$ $5.0 \mathrm{~Hz}, 1 \mathrm{H}), 6.98(\mathrm{~s}, 1 \mathrm{H}), 6.94(\mathrm{~d}, J=5.0 \mathrm{~Hz}, 1 \mathrm{H}), 4.13(\mathrm{q}, J=7.1 \mathrm{~Hz}$, $2 \mathrm{H}), 2.79(\mathrm{t}, J=7.6 \mathrm{~Hz}, 2 \mathrm{H}), 2.35(\mathrm{t}, J=7.5 \mathrm{~Hz}, 2 \mathrm{H}), 2.32(\mathrm{~s}, 3 \mathrm{H})$, $2.06(\mathrm{~m}, 2 \mathrm{H}), 1.25(\mathrm{t}, J=7.1 \mathrm{~Hz}, 3 \mathrm{H}) .{ }^{13} \mathrm{C} \mathrm{NMR}\left(126 \mathrm{MHz}, \mathrm{CDCl}_{3}\right)$ : $\delta 173.3,160.8,148.8,147.4,123.7,122.1,60.1,37.1,33.6,24.8,20.9$, 14.1. HRMS (ESI-TOF) $(\mathrm{m} / z):[\mathrm{M}+\mathrm{H}]^{+}$calcd for $\mathrm{C}_{12} \mathrm{H}_{18} \mathrm{NO}_{2}$ 208.1338 , found 208.1324 .

4-Methyl-2-(oxetan-3-yl)pyridine (14e). Ligand 4: 22 h, 49\% yield (from 2.0 equiv of 3-bromooxetane). Colorless oil. ${ }^{1} \mathrm{H}$ NMR (401 $\left.\mathrm{MHz}, \mathrm{CDCl}_{3}\right): \delta 8.47(\mathrm{~d}, J=5.0 \mathrm{~Hz}, 1 \mathrm{H}), 7.11(\mathrm{~s}, 1 \mathrm{H}), 7.01(\mathrm{~d}, J=5.0$ $\mathrm{Hz}, 1 \mathrm{H}), 5.06(\mathrm{dd}, J=8.4,5.8 \mathrm{~Hz}, 2 \mathrm{H}), 4.93(\mathrm{t}, J=7.5 \mathrm{~Hz}, 2 \mathrm{H}), 4.37-$ $4.30(\mathrm{~m}, 1 \mathrm{H}), 2.36(\mathrm{~s}, 3 \mathrm{H}) .{ }^{13} \mathrm{C}$ NMR $\left(101 \mathrm{MHz} \mathrm{CDCl}_{3}\right): \delta 160.1$, 149.2, 147.7, 122.9, 122.3, 76.9, 41.5, 20.9. HRMS (ESI-TOF) $(\mathrm{m} / z)$ : $[\mathrm{M}+\mathrm{H}]^{+}$calcd for $\mathrm{C}_{9} \mathrm{H}_{11} \mathrm{NO} 150.0919$, found 150.0914 .

2-Cyclopentyl-4-methylpyridine (14f). Ligand 4: $22 \mathrm{~h}, 49 \%$ yield (from 2.0 equiv of bromocyclopentane). Colorless oil. ${ }^{1} \mathrm{H}$ NMR (500 $\left.\mathrm{MHz}, \mathrm{CDCl}_{3}\right): \delta 8.38(\mathrm{~d}, J=5.0 \mathrm{~Hz}, 1 \mathrm{H}), 6.99(\mathrm{~s}, 1 \mathrm{H}), 6.89(\mathrm{~d}, J=5.0$ $\mathrm{Hz}, 1 \mathrm{H}), 3.16-3.06(\mathrm{~m}, 1 \mathrm{H}), 2.30(\mathrm{~s}, 3 \mathrm{H}), 2.09-2.03(\mathrm{~m}, 2 \mathrm{H}), 1.85-$ $1.80(\mathrm{~m}, 2 \mathrm{H}), 1.78-1.73(\mathrm{~m}, 2 \mathrm{H}), 1.71-1.66(\mathrm{~m}, 2 \mathrm{H}) .{ }^{13} \mathrm{C}$ NMR $\left(126 \mathrm{MHz}, \mathrm{CDCl}_{3}\right): \delta 165.2,148.7,147.0,122.4,121.8,47.7,33.4$, 25.7, 20.9. HRMS (ESI-TOF) $(\mathrm{m} / z):[\mathrm{M}+\mathrm{H}]^{+}$calcd for $\mathrm{C}_{11} \mathrm{H}_{16} \mathrm{~N}$ 162.1283 , found 162.1277 .

2-(6-Methoxypyridin-3-yl)ethyl Benzoate (14g). Ligand 4: $22 \mathrm{~h}$, $58 \%$ yield. Colorless oil. ${ }^{1} \mathrm{H}$ NMR $\left(401 \mathrm{MHz}, \mathrm{CDCl}_{3}\right): \delta 8.09(\mathrm{~d}, J=$ $2.3 \mathrm{~Hz}, 1 \mathrm{H}), 8.01(\mathrm{~m}, 2 \mathrm{H}), 7.55-7.50(\mathrm{~m}, 2 \mathrm{H}), 7.42(\mathrm{dd}, J=10.7,4.7$ $\mathrm{Hz}, 2 \mathrm{H}), 6.71(\mathrm{~d}, J=8.5 \mathrm{~Hz}, 1 \mathrm{H}), 4.48(\mathrm{t}, J=6.7 \mathrm{~Hz}, 2 \mathrm{H}), 3.92(\mathrm{~s}$, $3 \mathrm{H}), 3.00(\mathrm{t}, J=6.7 \mathrm{~Hz}, 2 \mathrm{H}) .{ }^{13} \mathrm{C}$ NMR $\left(101 \mathrm{MHz}, \mathrm{CDCl}_{3}\right): \delta 166.4$, $163.1,146.6,139.2,132.9,130.0,129.5,128.3,126.0,110.7,65.1,53.3$, 31.5. HRMS (ESI-TOF) $(\mathrm{m} / z):[\mathrm{M}+\mathrm{H}]^{+}$calcd for $\mathrm{C}_{15} \mathrm{H}_{16} \mathrm{NO}_{3}$ 258.1130 , found 258.1113 .

5-Cyclopentyl-2-methoxypyridine (14h). Ligand 4: 9 h, $82 \%$ yield. Colorless oil. ${ }^{1} \mathrm{H}$ NMR $\left(401 \mathrm{MHz}, \mathrm{CDCl}_{3}\right): \delta 8.02(\mathrm{~d}, J=2.4 \mathrm{~Hz}$, $1 \mathrm{H}), 7.44(\mathrm{dd}, J=8.5,2.4 \mathrm{~Hz}, 1 \mathrm{H}), 6.68(\mathrm{~d}, J=8.5 \mathrm{~Hz}, 1 \mathrm{H}), 3.91(\mathrm{~s}$, $3 \mathrm{H}), 2.96-2.88(\mathrm{~m}, 1 \mathrm{H}), 2.07-2.00(\mathrm{~m}, 2 \mathrm{H}), 1.84-1.75(\mathrm{~m}, 2 \mathrm{H})$, $1.73-1.62(\mathrm{~m}, 2 \mathrm{H}), 1.56-1.47(\mathrm{~m}, 2 \mathrm{H}) .{ }^{13} \mathrm{C}$ NMR $(101 \mathrm{MHz}$, $\left.\mathrm{CDCl}_{3}\right): \delta 162.5,144.9,137.4,134.0,110.3,53.1,42.4,34.4,25.3$. HRMS (ESI-TOF) $(m / z):[\mathrm{M}+\mathrm{H}]^{+}$calcd for $\mathrm{C}_{11} \mathrm{H}_{16} \mathrm{NO} 178.1232$, found 178.1217

Ethyl 4-(6-methoxypyridin-3-yl)butanoate (14i). Ligand 4: $20 \mathrm{~h}$, $67 \%$ yield. Colorless oil. ${ }^{1} \mathrm{H}$ NMR $\left(401 \mathrm{MHz}, \mathrm{CDCl}_{3}\right): \delta 7.97(\mathrm{~d}, J=$ $2.4 \mathrm{~Hz}, 1 \mathrm{H}), 7.41(\mathrm{dd}, J=8.5,2.4 \mathrm{~Hz}, 1 \mathrm{H}), 6.68(\mathrm{~d}, J=8.5 \mathrm{~Hz}, 1 \mathrm{H})$, $4.12(\mathrm{q}, J=7.1 \mathrm{~Hz}, 2 \mathrm{H}), 3.90(\mathrm{~s}, 3 \mathrm{H}), 2.57(\mathrm{t}, J=7.6 \mathrm{~Hz}, 2 \mathrm{H}), 2.31(\mathrm{t}$, $J=7.4 \mathrm{~Hz}, 2 \mathrm{H}), 1.95-1.87(\mathrm{~m}, 2 \mathrm{H}), 1.25(\mathrm{t}, J=7.1 \mathrm{~Hz}, 3 \mathrm{H}) .{ }^{13} \mathrm{C}$ $\operatorname{NMR}\left(101 \mathrm{MHz}, \mathrm{CDCl}_{3}\right): \delta 173.1,162.7,146.0,138.8,129.1,110.4$, $60.2,53.1,33.3,31.1,26.3,14.1$. HRMS (ESI-TOF) $(\mathrm{m} / z):[\mathrm{M}+\mathrm{H}]^{+}$ calcd for $\mathrm{C}_{12} \mathrm{H}_{18} \mathrm{NO}_{3}$ 224.1287, found 224.1272.

(6-Methoxypyridin-3-yl)methyl Pivalate (14j). Ligand 4: 17 h, 55\% yield (from chloromethyl pivalate). Colorless oil. ${ }^{1} \mathrm{H}$ NMR $(500 \mathrm{MHz}$, $\left.\mathrm{CDCl}_{3}\right): \delta 8.16(\mathrm{~s}, 1 \mathrm{H}), 7.57(\mathrm{dd}, J=8.4,2.5 \mathrm{~Hz}, 1 \mathrm{H}), 6.74(\mathrm{~d}, J=8.4$ $\mathrm{Hz}, 1 \mathrm{H}), 5.04(\mathrm{~s}, 2 \mathrm{H}), 3.94(\mathrm{~s}, 3 \mathrm{H}), 1.20(\mathrm{~s}, 9 \mathrm{H}) .{ }^{13} \mathrm{C}$ NMR $(126$ $\left.\mathrm{MHz}, \mathrm{CDCl}_{3}\right): \delta 178.3,164.1,146.8,139.0,124.8,110.8,63.4,54.1$,
38.7, 27.1. HRMS (ESI-TOF) $(m / z):[\mathrm{M}+\mathrm{H}]^{+}$calcd for $\mathrm{C}_{12} \mathrm{H}_{18} \mathrm{NO}_{3}$ 224.1287, found 224.1282

5-Cyclohexyl-2-fluoropyridine (14k). Ligand 4: $40 \mathrm{~h}, 53 \%$ yield. Pale yellow oil. ${ }^{1} \mathrm{H}$ NMR $\left(401 \mathrm{MHz}, \mathrm{CDCl}_{3}\right): \delta 8.04(\mathrm{~s}, 1 \mathrm{H}), 7.61(\mathrm{td}$, $J=8.2,2.4 \mathrm{~Hz}, 1 \mathrm{H}), 6.84(\mathrm{dd}, J=8.4,2.8 \mathrm{~Hz}, 1 \mathrm{H}), 2.59-2.48(\mathrm{~m}$, $1 \mathrm{H}), 1.86(\mathrm{~d}, J=8.3 \mathrm{~Hz}, 4 \mathrm{H}), 1.77(\mathrm{~d}, J=12.8 \mathrm{~Hz}, 1 \mathrm{H}), 1.44-1.34$ $(\mathrm{m}, 4 \mathrm{H}), 1.28-1.23(\mathrm{~m}, 1 \mathrm{H}) \cdot{ }^{13} \mathrm{C}$ NMR $\left(101 \mathrm{MHz}, \mathrm{CDCl}_{3}\right): \delta 162.1$ $(\mathrm{d}, J=237 \mathrm{~Hz}), 145.8(\mathrm{~d}, J=14.1 \mathrm{~Hz}), 140.6(\mathrm{~d}, J=5.0 \mathrm{~Hz}), 139.3(\mathrm{~d}$, $J=4.0 \mathrm{~Hz}), 109.1(\mathrm{~d}, J=37.4 \mathrm{~Hz}), 41.1,34.3,26.6,25.8 .{ }^{19} \mathrm{~F}$ NMR $\left(377 \mathrm{MHz}, \mathrm{CDCl}_{3}\right): \delta$ 59.6. HRMS (ESI-TOF) $(\mathrm{m} / z):[\mathrm{M}+\mathrm{H}]^{+}$ calcd for $\mathrm{C}_{11} \mathrm{H}_{15} \mathrm{FN}$ 180.1189, found 180.1186 .

3-Cyclohexylpyridine (14l). Ligand 4: $21 \mathrm{~h}, 64 \%$ yield. Pale yellow oil. ${ }^{1} \mathrm{H}$ NMR $\left(500 \mathrm{MHz}, \mathrm{CDCl}_{3}\right): \delta 8.49(\mathrm{~s}, 1 \mathrm{H}), 8.44(\mathrm{~d}, J=4.8 \mathrm{~Hz}$, $1 \mathrm{H}), 7.53(\mathrm{~d}, J=7.8 \mathrm{~Hz}, 1 \mathrm{H}), 7.22(\mathrm{dd}, J=7.8,4.8 \mathrm{~Hz}, 1 \mathrm{H}), 2.53(\mathrm{~m}$, $1 \mathrm{H}), 1.85(\mathrm{~m}, 4 \mathrm{H}), 1.76(\mathrm{~m}, 1 \mathrm{H}), 1.46-1.35(\mathrm{~m}, 4 \mathrm{H}), 1.30-1.21(\mathrm{~m}$, $1 \mathrm{H}) .{ }^{13} \mathrm{C}$ NMR $\left(126 \mathrm{MHz}, \mathrm{CDCl}_{3}\right): \delta 148.6,147.0,143.0,134.3$, 123.3, 41.9, 34.0, 26.5, 25.8. HRMS (ESI-TOF) $(\mathrm{m} / z):[\mathrm{M}+\mathrm{H}]^{+}$

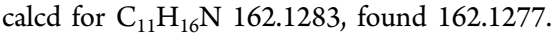

2-Fluoro-5-(oxetan-3-yl)pyridine (14m). Ligand 4: $15 \mathrm{~h}, 70 \%$ yield. White solid. Mp: $70-72{ }^{\circ} \mathrm{C}{ }^{1} \mathrm{H}$ NMR (500 MHz, $\mathrm{CDCl}_{3}$ ): $\delta 8.16$ (s, $1 \mathrm{H}), 8.00(\mathrm{t}, J=7.9 \mathrm{~Hz}, 1 \mathrm{H}), 7.00(\mathrm{dd}, J=8.4,2.6 \mathrm{~Hz}, 1 \mathrm{H}), 5.13(\mathrm{~m}$ $2 \mathrm{H}), 4.71(\mathrm{~m}, 2 \mathrm{H}), 4.29-4.23(\mathrm{~m}, 1 \mathrm{H}) .{ }^{13} \mathrm{C}$ NMR $(126 \mathrm{MHz}$, $\left.\mathrm{CDCl}_{3}\right): \delta 162.7(\mathrm{~d}, J=239 \mathrm{~Hz}), 146.0(\mathrm{~d}, J=15.1 \mathrm{~Hz}), 139.2(\mathrm{~d}, J=$ $7.6 \mathrm{~Hz}), 134.7(\mathrm{~d}, J=3.8 \mathrm{~Hz}) 109.7(\mathrm{~d}, J=37.8 \mathrm{~Hz}), 78.2,36.9 .{ }^{19} \mathrm{~F}$ NMR $\left(377 \mathrm{MHz}, \mathrm{CDCl}_{3}\right): \delta$ 61.9. HRMS (ESI-TOF) $(\mathrm{m} / z):[\mathrm{M}+$ $\mathrm{H}]^{+}$calcd for $\mathrm{C}_{8} \mathrm{H}_{9} \mathrm{FNO} 154.0668$, found 154.0658 .

5-sec-Butyl-2-methoxypyridine (14n). Ligand 4: $70 \mathrm{~h}, 58 \%$ yield (from 2 equiv of 2-bromobutane, $7 \mathrm{~mol} \%$ catalyst). Colorless oil. ${ }^{1} \mathrm{H}$ NMR (400 MHz, CDCl $): \delta 7.98(\mathrm{~d}, J=2.4 \mathrm{~Hz}, 1 \mathrm{H}), 7.42(\mathrm{dd}, J=$ $8.5,2.4 \mathrm{~Hz}, 1 \mathrm{H}), 6.71(\mathrm{~d}, J=8.5 \mathrm{~Hz}, 1 \mathrm{H}), 3.94(\mathrm{~s}, 3 \mathrm{H}), 2.58(\mathrm{qt}, J=$ $7.0 \mathrm{~Hz}, 1 \mathrm{H}), 1.67-1.49(\mathrm{~m}, 2 \mathrm{H}), 1.24(\mathrm{~d}, J=7.0 \mathrm{~Hz}, 3 \mathrm{H}), 0.84(\mathrm{t}, J=$ $7.4 \mathrm{~Hz}, 3 \mathrm{H}) .{ }^{13} \mathrm{C} \mathrm{NMR}\left(101 \mathrm{MHz}, \mathrm{CDCl}_{3}\right): \delta 162.8,145.4,137.3$, $135.3,110.6,53.4,38.4,31.1,21.9,12.2$. HRMS (ESI-TOF) $(\mathrm{m} / \mathrm{z})$ : $[\mathrm{M}+\mathrm{H}]^{+}$calcd for $\mathrm{C}_{10} \mathrm{H}_{16} \mathrm{NO} 166.1226$, found 166.1224.

(2-Methylpyridin-4-yl)methyl Pivalate (14o). Ligand 4: 17 h, $72 \%$ yield (from chloromethyl pivalate). Colorless oil. ${ }^{1} \mathrm{H}$ NMR $(500 \mathrm{MHz}$, $\left.\mathrm{CDCl}_{3}\right): \delta 8.48(\mathrm{~d}, J=5.0 \mathrm{~Hz}, 1 \mathrm{H}), 7.09(\mathrm{~s}, 1 \mathrm{H}), 7.05(\mathrm{~d}, J=5.0 \mathrm{~Hz}$, $1 \mathrm{H}), 5.08(\mathrm{~s}, 2 \mathrm{H}), 2.57(\mathrm{~s}, 3 \mathrm{H}), 1.27(\mathrm{~s}, 9 \mathrm{H}) .{ }^{13} \mathrm{C}$ NMR $(126 \mathrm{MHz}$, $\left.\mathrm{CDCl}_{3}\right): \delta 178.0,158.6,149.3,145.6,121.1,118.7,64.1,38.8,27.1$, 24.4. HRMS (ESI-TOF) $(\mathrm{m} / z):[\mathrm{M}+\mathrm{H}]^{+}$calcd for $\mathrm{C}_{12} \mathrm{H}_{18} \mathrm{NO}_{2}$ 208.1338, found 208.1331.

4-Cyclopentyl-2-methylpyridine (14p). Ligand 4: $8 \mathrm{~h}, 72 \%$ yield. Yellow oil. ${ }^{1} \mathrm{H}$ NMR $\left(500 \mathrm{MHz}, \mathrm{CDCl}_{3}\right): \delta 8.40(\mathrm{~s}, 1 \mathrm{H}), 7.02(\mathrm{~s}, 1 \mathrm{H})$, $6.98(\mathrm{~d}, J=4.3 \mathrm{~Hz}, 1 \mathrm{H}), 2.97-2.90(\mathrm{~m}, 1 \mathrm{H}), 2.53(\mathrm{~s}, 3 \mathrm{H}), 2.09-2.04$ $(\mathrm{m}, 2 \mathrm{H}), 1.85-1.77(\mathrm{~m}, 2 \mathrm{H}), 1.74-1.65(\mathrm{~m}, 2 \mathrm{H}), 1.61-1.54(\mathrm{~m}, 2 \mathrm{H})$. ${ }^{13} \mathrm{C}$ NMR $\left(126 \mathrm{MHz}, \mathrm{CDCl}_{3}\right): \delta 158.0,156.2,148.8,122.3,119.8$, 45.1, 33.8, 25.5, 24.3. HRMS (ESI-TOF) $(m / z):[\mathrm{M}+\mathrm{H}]^{+}$calcd for $\mathrm{C}_{11} \mathrm{H}_{16} \mathrm{~N} 162.1283$, found 162.1278 .

2-Methyl-4-(oxetan-3-yl)pyridine (14q). Ligand 2: $21 \mathrm{~h}, 56 \%$ yield (from 1.5 equiv of 3-bromooxetane, $5 \mathrm{~mol} \% \mathrm{NiI}_{2}$ ). Ligand $4: 21 \mathrm{~h}$, $56 \%$ yield (from 1.5 equiv of 3-bromooxetane). Yellow oil. ${ }^{1} \mathrm{H}$ NMR $\left(500 \mathrm{MHz}, \mathrm{CDCl}_{3}\right): \delta 8.48(\mathrm{~d}, J=5.0 \mathrm{~Hz}, 1 \mathrm{H}), 7.19(\mathrm{~s}, 1 \mathrm{H}), 7.13(\mathrm{~d}, J$ $=5.0 \mathrm{~Hz}, 1 \mathrm{H}), 5.08(\mathrm{dd}, J=8.2,6.2 \mathrm{~Hz}, 2 \mathrm{H}), 4.73(\mathrm{t}, J=6.3 \mathrm{~Hz}, 2 \mathrm{H})$, 4.18-4.13 (m, 1H), $2.57(\mathrm{~s}, 3 \mathrm{H}) .{ }^{13} \mathrm{C}$ NMR $\left(126 \mathrm{MHz} \mathrm{CDCl}_{3}\right): \delta$ 158.6, 150.5, 149.2, 121.3, 118.9, 77.4, 39.3, 24.1. HRMS (ESI-TOF) $(\mathrm{m} / z):[\mathrm{M}+\mathrm{H}]^{+}$calcd for $\mathrm{C}_{9} \mathrm{H}_{12} \mathrm{NO} 150.0919$, found 150.0906 .

2-(2-(2-Methylpyridin-4-yl)ethyl)isoindoline-1,3-dione (14r). Ligand 4: $9 \mathrm{~h}, 86 \%$ yield. White solid. Mp: $122-124{ }^{\circ} \mathrm{C}{ }^{1} \mathrm{H}$ NMR $\left(500 \mathrm{MHz}, \mathrm{CDCl}_{3}\right): \delta 8.39(\mathrm{~d}, J=4.8 \mathrm{~Hz}, 1 \mathrm{H}), 7.84(\mathrm{dd}, J=5.0,3.3$ $\mathrm{Hz}, 2 \mathrm{H}), 7.72(\mathrm{dd}, J=5.0,3.3 \mathrm{~Hz}, 2 \mathrm{H}), 7.06(\mathrm{~s}, 1 \mathrm{H}), 6.99(\mathrm{~d}, J=4.8$ $\mathrm{Hz}, 1 \mathrm{H}), 3.94(\mathrm{t}, J=7.6 \mathrm{~Hz}, 2 \mathrm{H}), 2.97(\mathrm{t}, J=7.6 \mathrm{~Hz}, 2 \mathrm{H}), 2.51(\mathrm{~s}$ $3 \mathrm{H}) .{ }^{13} \mathrm{C}$ NMR $\left(126 \mathrm{MHz}, \mathrm{CDCl}_{3}\right): \delta 168.0,158.6,149.2,147.0$, 134.0, 131.9, 123.6, 123.3, 121.1, 38.1, 33.7, 24.3. HRMS (ESI-TOF) $(\mathrm{m} / z):[\mathrm{M}+\mathrm{H}]^{+}$calcd for $\mathrm{C}_{16} \mathrm{H}_{15} \mathrm{~N}_{2} \mathrm{O}_{2}$ 267.1134, found 267.1119.

Ethyl 4-(2-Methylpyridin-4-yl)butanoate (14s). Ligand 2: $56 \mathrm{~h}$, $62 \%$ yield $\left(\mathrm{NiI}_{2}\right.$ was used in place of $\left.\mathrm{NiCl}_{2}(\mathrm{dme})\right)$. Ligand 4: $70 \mathrm{~h}$, $54 \%$ yield. Colorless oil. ${ }^{1} \mathrm{H}$ NMR $\left(401 \mathrm{MHz}, \mathrm{CDCl}_{3}\right): \delta 8.38(\mathrm{~d}, J=$ $5.0 \mathrm{~Hz}, 1 \mathrm{H}), 6.98(\mathrm{~s}, 1 \mathrm{H}), 6.92(\mathrm{~d}, J=5.0 \mathrm{~Hz}, 1 \mathrm{H}), 4.13(\mathrm{q}, J=7.1 \mathrm{~Hz}$, $2 \mathrm{H}), 2.61(\mathrm{~m}, 2 \mathrm{H}), 2.52(\mathrm{~s}, 3 \mathrm{H}), 2.32(\mathrm{t}, J=7.4 \mathrm{~Hz}, 2 \mathrm{H}), 1.99-1.92$ $(\mathrm{m}, 2 \mathrm{H}), 1.26(\mathrm{t}, J=7.1 \mathrm{~Hz}, 3 \mathrm{H}) .{ }^{13} \mathrm{C} \mathrm{NMR}\left(101 \mathrm{MHz}^{-C} \mathrm{CDCl}_{3}\right): \delta$ 
$172.9,158.2,150.5,148.9,123.3,120.9,60.2,34.2,33.3,25.3,24.1$, 14.1. HRMS (ESI-TOF) $(\mathrm{m} / z):[\mathrm{M}+\mathrm{H}]^{+}$calcd for $\mathrm{C}_{12} \mathrm{H}_{18} \mathrm{NO}_{2}$ 208.1338, found 208.1322.

2-(2-Methylpyridin-4-yl)ethyl Benzoate (14t). Ligand 2: 22 h, 61\% yield $\left(\mathrm{NiI}_{2}\right.$ was used in place of $\left.\mathrm{NiCl}_{2}(\mathrm{dme})\right)$. Ligand $4: 7 \mathrm{~h}, 93 \%$ yield. White solid. Mp: $48-50{ }^{\circ} \mathrm{C} .{ }^{1} \mathrm{H}$ NMR $\left(500 \mathrm{MHz}, \mathrm{CDCl}_{3}\right.$ ): $\delta 8.43$ (d, $J$ $=5.0 \mathrm{~Hz}, 1 \mathrm{H}), 7.99(\mathrm{~d}, J=7.2 \mathrm{~Hz}, 2 \mathrm{H}), 7.55(\mathrm{t}, J=7.4 \mathrm{~Hz}, 1 \mathrm{H}), 7.43$ $(\mathrm{t}, J=7.7 \mathrm{~Hz}, 2 \mathrm{H}), 7.08(\mathrm{~s}, 1 \mathrm{H}), 7.02(\mathrm{~d}, J=5.0 \mathrm{~Hz}, 1 \mathrm{H}), 4.55(\mathrm{t}, J=$ $6.7 \mathrm{~Hz}, 2 \mathrm{H}), 3.03(\mathrm{t}, J=6.7 \mathrm{~Hz}, 2 \mathrm{H}), 2.54(\mathrm{~s}, 3 \mathrm{H}) .{ }^{13} \mathrm{C}$ NMR $(126$ $\left.\mathrm{MHz}, \mathrm{CDCl}_{3}\right): \delta 166.2,158.4,149.1,147.1,133.0,129.9,129.4,128.3$, 123.7, 121.2, 64.0, 34.4, 24.2. HRMS (ESI-TOF) $(m / z):[\mathrm{M}+\mathrm{H}]^{+}$ calcd for $\mathrm{C}_{15} \mathrm{H}_{16} \mathrm{NO}_{2}$ 242.1181, found 242.1163.

\section{ASSOCIATED CONTENT}

\section{(S) Supporting Information}

The Supporting Information is available free of charge on the ACS Publications website at DOI: 10.1021/acs.joc.7b01334.

${ }^{1} \mathrm{H}$ and ${ }^{13} \mathrm{C}$ NMR spectra for all new compounds (PDF)

\section{AUTHOR INFORMATION}

\section{Corresponding Authors}

*E-mail: eric.hansen@pfizer.com.

*E-mail: daniel.weix@rochester.edu.

\section{ORCID $\odot$}

Eric C. Hansen: 0000-0002-5057-4577

Daniel J. Weix: 0000-0002-9552-3378

\section{Author Contributions}

${ }^{\| E . C . H . ~ a n d ~ C . L . ~ c o n t r i b u t e d ~ e q u a l l y . ~}$

Notes

The authors declare no competing financial interest.

\section{ACKNOWLEDGMENTS}

Research reported in this publication was supported in part by the National Institute of General Medical Sciences of the National Institutes of Health under Award No. R01GM097243 (D.J.W.). This work was influenced by the ongoing efforts of the Non-Precious Metal Catalysis Alliance between Pfizer, Boehringer-Ingelheim, Abbvie, and Asymchem. We thank James Gage (Asymchem) for useful discussions and Don Batesky (University of Rochester) for synthesis of key ligand intermediates.

\section{REFERENCES}

(1) (a) Vitaku, E.; Smith, D. T.; Njardarson, J. T. J. Med. Chem. 2014, 57, 10257-10274. (b) Slagt, V. F.; de Vries, A. H. M.; de Vries, J. G.; Kellogg, R. M. Org. Process Res. Dev. 2010, 14, 30-47. (c) Badenock, J. C.; Gribble, G. W. Adv. Heterocycl. Chem. 2016, 120, 99-136.

(2) (a) Manolikakes, G. Comp. Org. Syn. II 2014, 3, 392-464. (b) Bull, J. A.; Mousseau, J. J.; Pelletier, G.; Charette, A. B. Chem. Rev. 2012, 112, 2642-2713. (c) Schlosser, M.; Mongin, F. Chem. Soc. Rev. 2007, 36, 1161-1172. (d) Slagt, V. F.; de Vries, A. H. M.; de Vries, J. G.; Kellogg, R. M. Org. Process Res. Dev. 2010, 14, 30-47. (e) Sherry, B. D.; Fürstner, A. Acc. Chem. Res. 2008, 41, 1500-1511. (f) Ila, H.; Baron, O.; Wagner, A. J.; Knochel, P. Chem. Commun. 2006, 583-593 The most general approaches involve either pyridylzinc or -tin reagents with alkyl halides or pyridyl halides with alkylaluminum, -magnesium, or -zinc reagents. See ref $6 \mathrm{~b}$ for a good overview as well as specific examples in refs $15-17$..

(3) (a) Duncton, M. A. J. MedChemComm 2011, 2, 1135-1161. (b) O'Hara, F.; Blackmond, D. G.; Baran, P. S. J. Am. Chem. Soc. 2013, 135, 12122-12134.

(4) (a) Dong, Z.; Ren, Z.; Thompson, S. J.; Xu, Y.; Dong, G. Chem. Rev. 2017, DOI: 10.1021/acs.chemrev.6b00574. (b) Lewis, J. C.; Bergman, R. G.; Ellman, J. A. J. Am. Chem. Soc. 2007, 129, 5332-5333. (c) Nakao, Y.; Yamada, Y.; Kashihara, N.; Hiyama, T. J. Am. Chem. Soc. 2010, 132, 13666-13668. (d) Guan, B.-T.; Hou, Z. J. Am. Chem. Soc. 2011, 133, 18086-18089. (e) Ryu, J.; Cho, S. H.; Chang, S. Angew. Chem., Int. Ed. 2012, 51, 3677-3681.

(5) (a) Gu, J.; Wang, X.; Xue, W.; Gong, H. Org. Chem. Front. 2015, 2, 1411-1421. (b) Weix, D. J. Acc. Chem. Res. 2015, 48, 1767-1775. (c) Knappke, C. E. I.; Grupe, S.; Gaertner, D.; Corpet, M.; Gosmini, C.; Jacobi von Wangelin, A. Chem. - Eur. J. 2014, 20, 6828-6842. (d) Everson, D. A.; Weix, D. J. J. Org. Chem. 2014, 79, 4793.

(6) Heteroaryl halides with alkyl halides and psuedohalides: (a) Wang, S.; Qian, Q.; Gong, H. Org. Lett. 2012, 14, 3352-3355. (b) Everson, D. A.; Buonomo, J. A.; Weix, D. J. Synlett 2014, 25, 233238. (c) Molander, G. A.; Traister, K. M.; O’Neill, B. T. J. Org. Chem. 2014, 79, 5771-5780. (d) Liu, H.; Liang, Z.; Qian, Q.; Lin, K. Synth. Commun. 2014, 44, 2999-3007. (e) Molander, G. A.; Traister, K. M.; O’Neill, B. T. J. Org. Chem. 2015, 80, 2907-2911. (f) Cai, D.-J.; Lin, P.-H.; Liu, C.-Y. Eur. J. Org. Chem. 2015, 2015, 5448-5452.

(7) (a) Kadunce, N. T.; Reisman, S. E. J. Am. Chem. Soc. 2015, 137, 10480-10483. (b) Poremba, K. E.; Kadunce, N. T.; Suzuki, N.; Cherney, A. H.; Reisman, S. E. J. Am. Chem. Soc. 2017, 139, 56845687.

(8) A closely related approach is the Lipshutz-Negishi coupling in water. This process has been applied to pyridine cores with promising results: (a) Krasovskiy, A.; Duplais, C.; Lipshutz, B. J. Am. Chem. Soc. 2009, 131, 15592-15593. (b) Krasovskiy, A.; Thomé, I.; Graff, J.; Krasovskaya, V.; Konopelski, P.; Duplais, C.; Lipshutz, B. H. Tetrahedron Lett. 2011, 52, 2203-2205. (c) Bhonde, V. R.; O’Neill, B. T.; Buchwald, S. L. Angew. Chem., Int. Ed. 2016, 55, 1849-1853.

(9) For a photoredox approach that successfully coupled secondary alkyl bromides with a variety of heteroaryl halides, see: Zhang, P.; Le, C. C.; MacMillan, D. W. C. J. Am. Chem. Soc. 2016, 138, 8084-8087.

(10) (a) Zuo, Z.; Ahneman, D.; Chu, L.; Terrett, J.; Doyle, A. G.; MacMillan, D. W. C. Science 2014, 345, 437-440. (b) Tellis, J. C.; Primer, D. N.; Molander, G. A. Science 2014, 345, 433-436. (c) Shields, B. J.; Doyle, A. G. J. Am. Chem. Soc. 2016, 138, 1271912722. (d) Shaw, M. H.; Shurtleff, V. W.; Terrett, J. A.; Cuthbertson, J. D.; MacMillan, D. W. C. Science 2016, 352, 1304-1308.

(11) (a) Cornella, J.; Edwards, J. T.; Qin, T.; Kawamura, S.; Wang, J.; Pan, C.-M.; Gianatassio, R.; Schmidt, M. A.; Eastgate, M. D.; Baran, P. S. J. Am. Chem. Soc. 2016, 138, 2174-2177. (b) Qin, T.; Cornella, J.; Li, C.; Malins, L. R.; Edwards, J. T.; Kawamura, S.; Maxwell, B. D.; Eastgate, M. D.; Baran, P. S. Science 2016, 352, 801-805.

(12) Hansen, E. C.; Pedro, D. J.; Wotal, A. C.; Gower, N. J.; Nelson, J. D.; Caron, S.; Weix, D. J. Nat. Chem. 2016, 8, 1126-1130.

(13) Although 4 has not been used as a ligand, it has been used as a synthetic intermediate towards other ligands: (a) Mauro, M.; Aliprandi, A.; Cebrian, C.; Wang, D.; Kubel, C.; De Cola, L. Chem. Commun. 2014, 50, 7269-7272. (b) Septiadi, D.; Aliprandi, A.; Mauro, M.; De Cola, L. RSC Adv. 2014, 4, 25709-25718. (c) Sanning, J.; Ewen, P. R.; Stegemann, L.; Schmidt, J.; Daniliuc, C. G.; Koch, T.; Doltsinis, N. L.; Wegner, D.; Strassert, C. A. Angew. Chem., Int. Ed. 2015, 54, 786-791.

(14) (a) Everson, D. A.; Shrestha, R.; Weix, D. J. J. Am. Chem. Soc. 2010, 132, 920-921. (b) Everson, D. A.; Jones, B. A.; Weix, D. J. J. Am. Chem. Soc. 2012, 134, 6146-6159. (c) Biswas, S.; Weix, D. J. J. Am. Chem. Soc. 2013, 135, 16192-16197. (d) Johnson, K. A.; Biswas, S.; Weix, D. J. Chem. - Eur. J. 2016, 22, 7399-7402.

(15) (a) Sibille, S.; Nedelec, J. Y.; Perichon, J. Electroorg. Synth. 1991, 361-367. (b) Borjesson, M.; Moragas, T.; Martin, R. J. Am. Chem. Soc. 2016, 138, 7504-7507. (c) Tollefson, E. J.; Erickson, L. W.; Jarvo, E. R. J. Am. Chem. Soc. 2015, 137, 9760-9763.

(16) (a) Flohr, A.; Groebke, Z. K.; Kuhn, B.; Lerner, C.; Rudolph, M.; Schaffhauser, H. (F. Hoffmann-La Roche Ag; Hoffmann-La Roche Inc.) Triazolo compounds as pde10 inhibitors. WO Patent Appl. 178572A1, 2013;. (b) Troxler, T.; Hurth, K.; Schuh, K.-H.; Schoeffter, P.; Langenegger, D.; Enz, A.; Hoyer, D. Bioorg. Med. Chem. Lett. 2010, 20, 1728-1734. (c) Lu, T.; Alexander, R.; Connors, R. W.; Cummings, M. D.; Galemmo, R. A.; Hufnagel, H. R.; Johnson, D. L.; Khalil, E.; Leonard, K. A.; Markotan, T. P.; Maroney, A. C.; Sechler, J. L.; 
Travins, J. M.; Tuman, R. W. (Janssen Pharmaceutica, N. V.) Triazolopyridazines as tyrosine kinase modulators. WO Patent WO2007/75567, 2007.

(17) (a) Furuyama, H.; Kurihara, H.; Furuya, K.; Terao, T.; Sekine, S.; Nakagawa, D. (FUJIFILM Corporation). 1,5-naphthyridine derivative or salt thereof. Eur. Patent Appl. 2727920A1, 2014.

(b) Basinger, J.; Breitenbucher, J.; Freestone, G.; Gupta, V.; Kaplan, A.; Mak, C.-C.; Pratt, B.; Santora, V.; Sengupta, D.; Valdez, L. (Dart Neuroscience LLC). Substituted naphthyridine and quinoline compounds as mao inhibitors. US Patent Appl. US20140275548A1, 2014.

(18) Nagai, K.; Baba, M.; Fujioka, S.; Nagasawa, K.; Takahashi, H.; Kondoh, E.; Sogo, S.; Tanaka, K.; Itoh, Y. (Sato Pharmaceutical Co., Ltd.). Difluoromethylene compound. Eur. Patent Appl. EP2878594A1, 2015.

(19) (a) Dreher, S.; Dormer, P.; Sandrock, D.; Molander, G. J. Am. Chem. Soc. 2008, 130, 9257-9259. (b) Han, C.; Buchwald, S. J. Am. Chem. Soc. 2009, 131, 7532-7533.

(20) Anka-Lufford, L. L.; Huihui, K. M. M.; Gower, N. J.; Ackerman, L. K. G.; Weix, D. J. Chem. - Eur. J. 2016, 22, 11564-11567.

(21) For a recent study on related $N$-aryl-1-(pyridin-2-yl) methanimine ligands showing stark differences between these nonsymmetrical ligands and bipyridine ligands, see: Biewer, C.; Hamacher, C.; Kaiser, A.; Vogt, N.; Sandleben, A.; Chin, M. T.; Yu, S.; Vicic, D. A.; Klein, A. Inorg. Chem. 2016, 55, 12716-12727. 